\title{
miR675 upregulates long noncoding RNA H19 through activating EGR1 in human liver cancer
}

\author{
Haiyan Li ${ }^{1}$, Jiao $\mathrm{Li}^{2}$, Song Jia ${ }^{2}$, Mengying Wu${ }^{1}$, Jiahui An ${ }^{1}$, Qidi Zheng ${ }^{1}$, Wei Zhang ${ }^{1}$ \\ and Dongdong Lu ${ }^{1}$ \\ ${ }^{1}$ School of Life Science and Technology, Tongji University, Shanghai, China \\ ${ }^{2}$ School of Medicine, Tongji University, Shanghai, China \\ Correspondence to: Dongdong LU, email: ludongdong@tongji.edu.cn \\ Keywords: microRNA675, lincRNA H19, early growth response protein 1, pyruvate kinase M2, hepatoma \\ Received: May 19, $2015 \quad$ Accepted: August 20, $2015 \quad$ Published: September 10, 2015
}

This is an open-access article distributed under the terms of the Creative Commons Attribution License, which permits unrestricted use, distribution, and reproduction in any medium, provided the original author and source are credited.

\section{ABSTRACT}

microRNAs (miRNAs) are short non-coding RNAs that are involved in posttranscriptional regulation of gene expression in multicellular organisms by affecting both the stability and translation of mRNAs. miR675, embedded in H19's first exon , had been linked to the development of human cancers. Herein, we demonstrate miR675 overexpression promotes and silencing miR675 attenuated liver cancer cell growth in vitro and in vivo. Mechanistically, miR675 inhibits the heterochromatin1 isoform HP1a expression in human liver cancer cells which causes a dramatically decrease of the total histone $\mathrm{H} 3$ lysine 9 trimethylation (H3K9me3), histone H3 lysine 27 trimethylation (H3K27me3) and a increase of histone H3 lysine 27 acetylation(H3K27AC).Notably, a significant reduction of the H3K9me3 and H3K27me3 and the increment of $\mathrm{H} 3 \mathrm{~K} 27 \mathrm{AC}$ occupancy on the promoter region of EGR1 triggers EGR1 transcription, translation, sumoylation and activation which upregulates lincRNA H19. Strikingly, H19 may induce and activate tumor-specific pyruvate kinase M2 (PKM2) which is essential for the Warburg effect in its dimer and for gene expression in its teramer during tumorigenesis. Our results imply that miR675 is involved in the epigenetic regulation of $\mathrm{H} 3 \mathrm{~K} 9 \mathrm{me} 3, \mathrm{H} 3 \mathrm{k} 27 \mathrm{me} 3$ and $\mathrm{H} 3 \mathrm{~K} 27 \mathrm{Ac}$ for gene expression and function during hepatocarcinogenesis (e.g.C-myc,Pim1,Ras,CyclinD1,RB1).These findings sheds light on the significance of miR675-HP1a-EGR1-H19-PKM2 cascade signaling pathway in liver cancer.

\section{INTRODUCTION}

Primary liver cancer are an increasing global health problem, with hepatocellular carcinoma (HCC) now being the third leading cause of cancer-related mortality worldwide [1]. MicroRNAs are potent regulators of gene expression and modulate multiple cellular processes including proliferation, differentiation, apoptosis and tumorigenesis. miR675, a miRNA, embedded in H19's first exon, is expressed exclusively in the placenta. Overexpression of miR-675 in a range of embryonic and extra-embryonic cell lines results in their reduced proliferation [2]. miR675 was shown to up-regulate the essential cartilage matrix component COL2A1, and overexpression of miR-675 rescued COL2A1 levels in H19- or SOX9-depleted cells [3]. Cadherin 11 in fibroblasts and keratinocytes is a target of miR-675, and could be involved in melanogenesis through the induction of N-cadherin during epithelial-mesenchymal transition [4]. H19 regulates glioma development by deriving miR-675 which modulated Cadherin 13 expression by directly targeting the binding site within the 3' UTR [5].In addition, H19 maintain hematopoietic stem cell repopulating ability through a miR-675-IGFR signaling circuit [6]. However, others resports also showed the different functions of miR675. For examples, miR675 was significantly downregulated in the metastatic prostate cancer cell and directly bound with 3'UTR of transforming growth factor $\beta$ induced protein (TGFBI, an extracellular matrix protein involved in cancer metastasis) mRNA to repress its translation [7]. H19 gene could 
inhibit human trophoblast cell proliferation via encoding miR675 that targeted NOMO1 and interferes with Nodal signaling [8]. Intriguingly, miR675 helps in discriminating adrenocortical carcinomas (ACCs) from adrenocortical adenomas (ACAs) [9]. However, the exact roles of mature miR-675 in hepatocarcinogenesis have not been identified.

Heterochromatin causes epigenetic repression that can be transmitted through multiple cell divisions. Heterochromatic-silencing factors preclude histone turnover to promote silencing and inheritance of repressive chromatin [10]. HP1 $\alpha$ is an essential protein critical for heterochromatin assembly and regulation. HP1 $\alpha$ nucleates with high affinity independently of $\mathrm{H} 3 \mathrm{~K} 9 \mathrm{me}$ in promoters of active genes and then spreads via $\mathrm{H} 3 \mathrm{~K} 9$ methylation and transient looping contacts with those H3K9me target sites [11].HP1 mediates the recognition and destruction of heterochromatic RNA transcripts [12]. Strikingly, HP1 promotes tumor suppressor BRCA1 functions during the DNA damage response [13].DNA double-strand breaks promote methylation of histone $\mathrm{H} 3$ on lysine 9 and transient formation of repressive chromatin [14]. Recently, a research indicate the hyperacetylation of H3K9 at EGR1 binding sites in promoter region II of the GDNF gene can up-regulate the binding of EGR 1 to increase GDNF gene transcription in glioma cells [15]. Arf-EGR1-C/EBP $\beta$ axis as an important determinant of cellular responses (senescence or transformation) to oncogenic Ras signaling [16]. The loss of Tp53 activity in cooperation with EGR1 and Apc haploinsufficiency creates an environment that is permissive for malignant transformation and the development of AML [17]. 2'-Benzoyloxycinnamaldehyde (BCA) induces prostate cancer cell death via EGR1 upregulation and nuclear translocalization, followed by activation of proapoptotic target genes [18].

Increasing evidence suggests that non-coding RNAs have multiple important roles in transcriptional regulation, and also contribute to the expansion of genome complexity. The long noncoding RNA H19 has been implicated in development and growth control and is associated with human genetic disorders and cancer. $\mathrm{H} 19$ has been recently characterized as an oncogenic lncRNA in some tumors. An HIV-encoded antisense long noncoding RNA epigenetically regulates viral transcription and overexpression of H19 significantly increased the sphere-forming capacity [19]. H19 RNA expression is to regulate the expression of IGF2 (Insulin Growth Factor 2) [20].The methylated paternal H19 allele replicates early in the $\mathrm{S}$ phase while the hypomethylated maternal allele replicates later, and the later-replicating maternal H19 allele is CTCF-bound [21].Knock-down of H19 lead to increased polyploidization of mesenchymal stem cells, and induced polyploidy resulted in reduced expression of H19, providing a direct link between H19 expression and the amount of DNA within the cell [22]. H19 is expressed at high levels in cancer cells and increased H19 expression is found in some cancers.e.g. adrenocortical neoplasms, choriocarcinomas, hepatocellular carcinomas, bladder cancers, esophageal cancer and lung cancer [23, 24, 25, 26, 27].Cells expressing H19 are able to form bigger colonies in soft agar and subcutaneous injection of H19 into mice promoted tumor progression [28]. Downregulation of H19 in breast and lung cancer cells decreases their clonogenicity and anchorage-dependent growth [29]. The effect of H19 in gastric cancer is mediated by the direct upregulation of ISM1 and the indirect suppression of CALN1 expression via miR-675 [30]. In HCC, the expression of H19 and IGF2 usually changes from monoallelic to biallelic. In in vitro studies, culturing HCC cell lines in hypoxic condition upregulated H19 expression [31]. In addition, H19 is positively correlated with the presence of steroid receptors, uPar, c-src kinase, tyrosine kinase 2 mitogen-activated protein kinase kinase, tyrosine kinase 2, c-jun, JNK1 [32]. Intriguingly, Maternal imprinting at the H19-Igf2 locus maintains adult haematopoietic stem cell quiescence [33]. The H19 expression is able to function as a cascade activator of trophoblast lineage commitment possibly by overriding the Oct3/4 action in ESCs [34].H19 modulates let-7 availability by acting as a molecular sponge [35]. Strikingly, H19 depletion results in impaired insulin signaling and decreased glucose uptake [36].Notably, silencing Mineral dust-induced gene (mdig) increased the level of $\mathrm{H} 3 \mathrm{~K} 9 \mathrm{me} 3$ in the promoter region of $\mathrm{H} 19$ but also attenuated the transcription of H19 long non-coding RNA [37]. Intriguingly, histone H1.3 overexpression leads to increase occupancy of $\mathrm{H} 1.3$ at the $\mathrm{H} 19$ regulator region encompassing the imprinting control region (ICR) so that H1.3 dramatically inhibits H19 expression, which contributes to the suppression of epithelial ovarian carcinogenesis [38].

Abnormal metabolism and sustained proliferation are hallmarks of cancer. Pyruvate kinase M2 (PKM2) is a metabolic enzyme that plays important roles in both processes. PKM2 is subjected to a complex regulation by both oncogenes and tumour suppressors, which allows for a fine-tone regulation of PKM2 activity. PKM2 possesses protein tyrosine kinase activity and plays a role in modulating gene expression and thereby contributing to tumorigenesis [39]. While dimeric PKM2 diverts glucose metabolism towards anabolism through aerobic glycolysis, tetrameric PKM2 promotes the flux of glucose-derived carbons. Equilibrium of the PKM2 dimers and tetramers is critical for tumorigenesis. PKM2 promotes glucose metabolism and cell growth in gliomas through a mechanism involving a let-7a/cMyc/hnRNPA1 feedback loop [40].JMJD5, a Jumonji C domain-containing dioxygenase, interacts directly with pyruvate kinase muscle isozyme (PKM)2 to modulate metabolic flux in cancer cells. The JMJD5-PKM2 interaction resides at the intersubunit interface region of 
PKM2, which hinders PKM2 tetramerization and blocks pyruvate kinase activity [41]. LPS induces expression of the key metabolic regulator PKM2. PKM2 is therefore a critical determinant of macrophage activation by LPS, promoting the inflammatory response [42]. The binding of PKM2 with TGF- $\beta$-induced factor homeobox 2 (TGIF2) recruits histone deacetylase 3 to the E-cadherin promoter sequence, with subsequent deacetylation of histone $\mathrm{H} 3$ and suppression of E-cadherin transcription , leading to epithelial-mesenchymal transition [43]. It is long known that PKM2 promotes tumor angiogenesis by increasing endothelial cell proliferation, migration, and cell-ECM adhesion. Only the dimeric PKM2 possess the activity in promoting tumor angiogenesis [44].The PKM2 knockdown-resistant cells were further subdivided into less glycolytic and more (glycolysis branch pathway-dependent) glycolytic groups [45]. Recently, PKM2 was shown to have protein kinase activity phosphorylating histone $\mathrm{H} 3$ and promoting cancer cell proliferation [46]. Regulation of PKM2 activity supports the different metabolic requirements of proliferating and nonproliferating tumor cells [47].Strikingly, tissuespecific isoform switch and DNA hypomethylation of the pyruvate kinase PKM gene in human cancers [48]. PKM2 is instrumental in both aerobic glycolysis and gene transcription. PKM2 regulates G1-S phase transition by controlling cyclin D1 expression. PKM2 binds to the spindle checkpoint protein Bub3 during mitosis and phosphorylates Bub3 at Y207. Moreover, the level of Bub3 Y207 phosphorylation correlated with histone H3S10 phosphorylation in human glioblastoma specimens and with glioblastoma prognosis [49].

In this report, we demonstrate miR675 is involved in the epigenetic regulation of $\mathrm{H} 3 \mathrm{~K} 9 \mathrm{me} 3$, H3K27me3, H3K27Ac for gene expression during hepatocarcinogenesis. miR675 overexpression promotes liver cancer cell growth in vitro and in vivo. miR675 upregulates lincRNA H19. Strikingly, H19 induces and activates tumor-specific pyruvate kinase M2 (PKM2) which is essential for the warburg effect in its dimer and for gene expression in its teramer during tumorigenesis. These findings sheds light on the significance of miR675HP1 $\alpha$-EGR1-H19-PKM2 cascade signaling pathway in cancer cells.

\section{RESULTS}

\section{miR675 promotes liver cancer cells malignant proliferation}

To address whether the miR675 alters primary liver cancer cells malignant proliferation capacity, we first established the stable human liver cancer cell lines (Hep3B) transfected with pCMV-miR, pCMV-miR675,
pGFP-V-RS, pGFP-V-RS-miR675 respectively. We selected the GFP positive cell for screening miR675 overexpression or knockdown stable cell lines (Figure $1 \mathrm{~A}$ a, left and Figure $1 \mathrm{~B}$ a, right ). We confirmed mature miR675 expression using real-time RT-PCR and the results showed that mature miR675 was significantly overexpressed in pCMV6-miR675 transfected Hep3B compared with control $(P<0.01)$ and the expression of $3 \#$ clone is slight higher compared to 6\# (Figure 1Aa, right , 3\#\&6\#), while mature miR675 was significantly knocked down in pGFP-V-miR675 transfected Hep3B compared the control $(P<0.01)$ ( (Figure 1Ba, left). At the first time, we detected these cells proliferation capacity in vitro using CCK8. As shown in Figure $1 \mathrm{Ab}$, mature miR675 overexpression promoted Hep3B proliferation (the $2^{\text {nd }}$ day $\&$ the $3^{\text {rd }}$ day , $P<0.01$ ). Strikingly, the growth from $3 \#$ clone was significant faster than that from $6 \#$ ( $P$ $<0.01)$. On the contrast, mature miR675 knockdown inhibited Hep3B proliferation (the $2^{\text {nd }}$ day $\&$ the $3^{\text {rd }}$ day , $P<0.01$ ) (Figure 1Bb). The colony-formation rate was significantly increased in mature miR675 overexpressed Hep3B compared to control Hep3B (37.63 $\pm 2.18 \%$ vs $9.93 \pm 1.03 \%, P<0.01$ ) (Figure $1 \mathrm{Ab}$ ). In contrast, the plate colony-formation rate was significantly decreased in mature miR675 knocked down Hep3B compared to control Hep3B $(16.3 \pm 4.26 \%$ vs $8.63 \pm 0.38 \%, P<0.01)$ (Figure 1Bc).

Further on, we slected the human liver cancer cell lines HepG2 for in vitro tumorigenesis test. Our results showed that mature miR675 was significantly overexpressed in pCMV-miR675 transfected HepG2 compared to control $(P<0.01)$, while mature miR675 was significantly knocked down in pGFP-V-RSmiR675 transfected HepG2 compared to the control $(P$ $<0.01$ ) (Figure 1Ca). Mature miR675 overexpression promoted and miR675 knockdown inhibited HepG2 proliferation $(P<0.01)$ (Figure $1 \mathrm{Cb})$, colony formation ability $(75.63 \pm 11.74 \%$ versus $36.93 \pm 4.5 \% ; 15.46 \pm 4.35 \%$ versus $40.2 \pm 10.63 \%$, respectively, $P<0.01$ ) (Figure $1 \mathrm{Cc}), \mathrm{Brdu}$ position rate $(83.33 \pm 9.22 \%$ versus $41.0 \pm 10.35 \% ; 22.21 \pm 3.90 \% \quad$ versus $\quad 48.03 \pm 7.24 \%$, respectively, $P<0.01$ ) (Figure $1 \mathrm{Cd}$ ). Collectively, these results suggest that miR675 promotes the liver cancer cells malignant proliferation.

\section{miR675 accelerates liver cancer cells growth in vivo}

Given that the miR675 promotes liver cancer cells malignant proliferation, we further consider to identity the effect of miR675 on hepatocarcinogenesis in vivo. The Hep3B stable cell lines with altered expression of mature miR675 were injected subcutaneously into Balb/C nude mice. There were 36 cases of Balb/C mice in first animal tumorigenesis test in vivo. As shown in Figure 

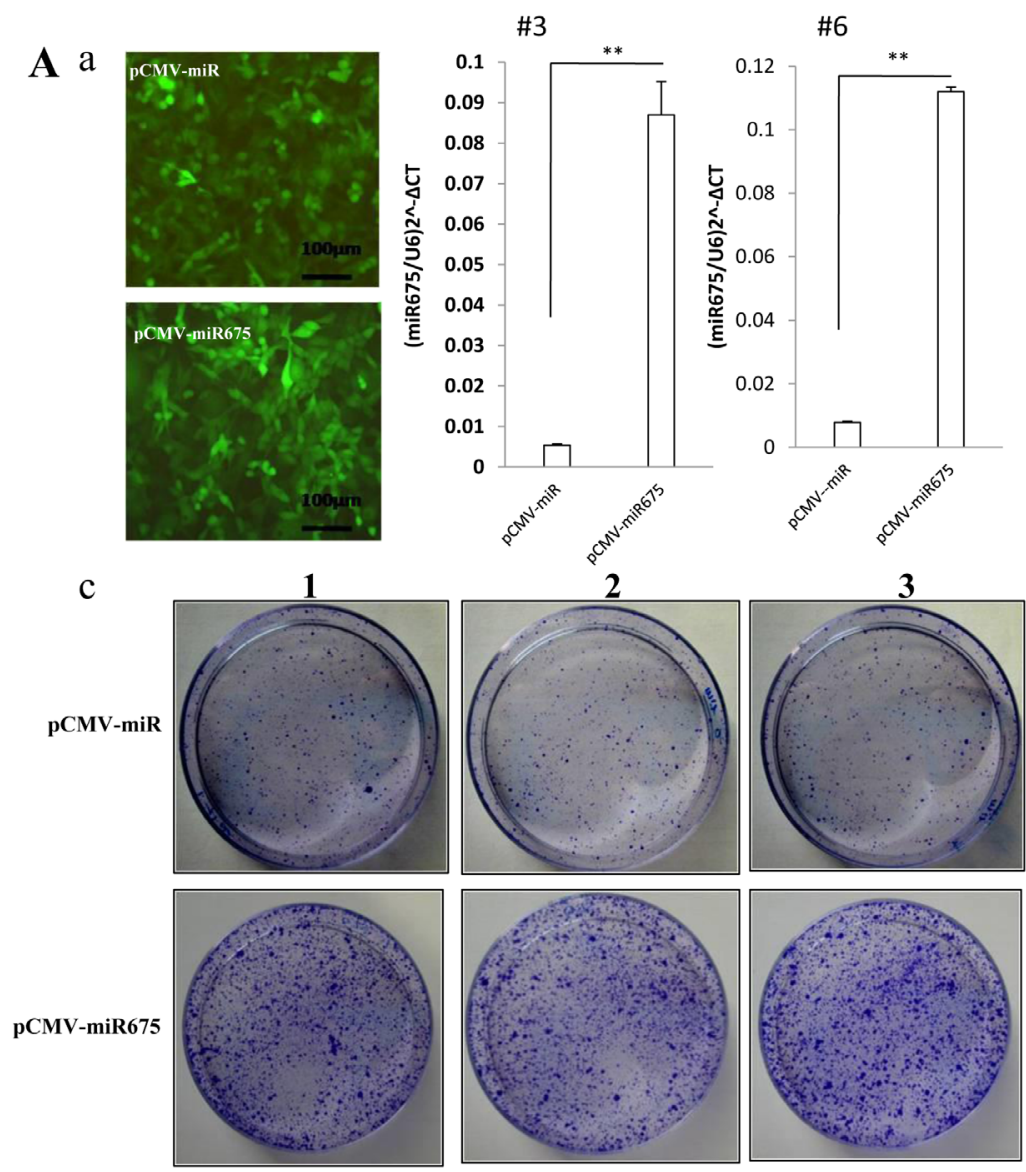

B

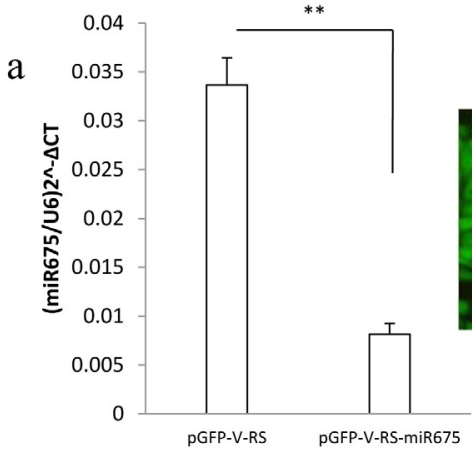

$\mathrm{c}$
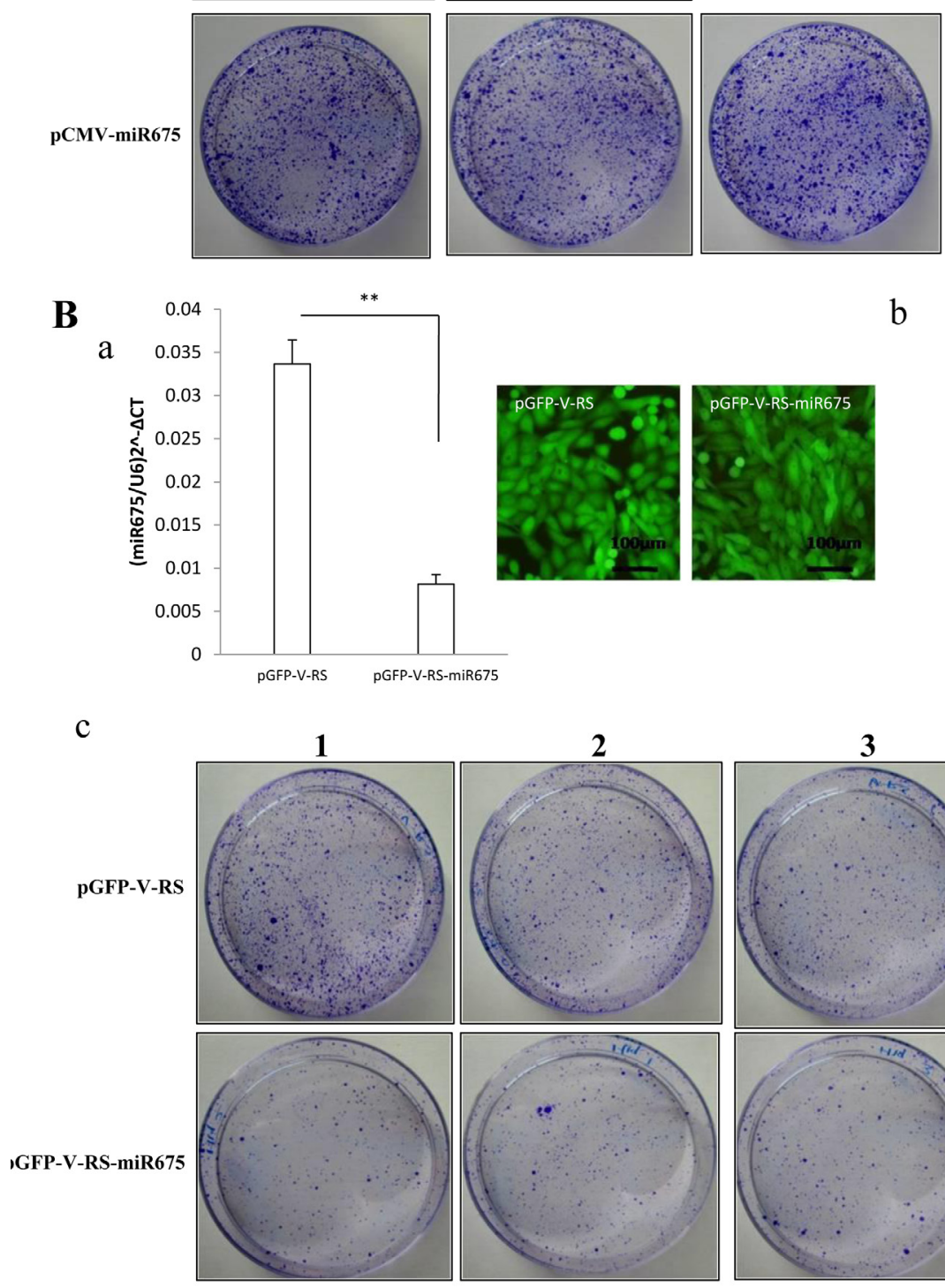

2

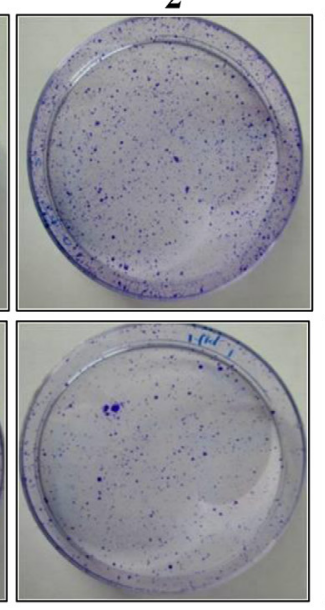

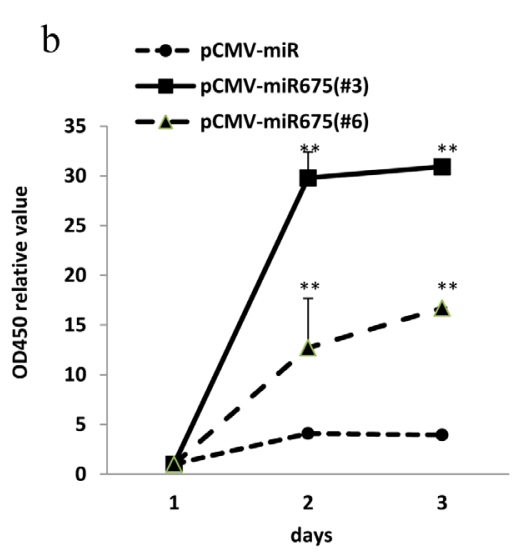

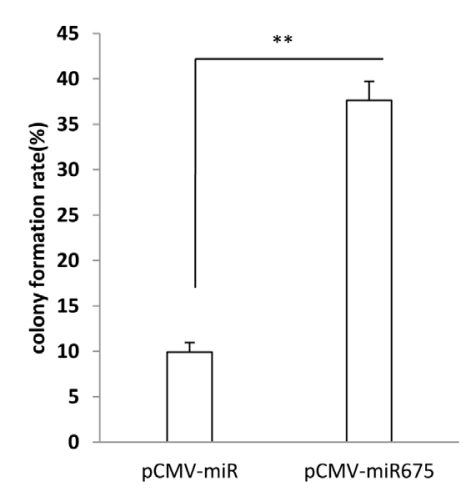

b

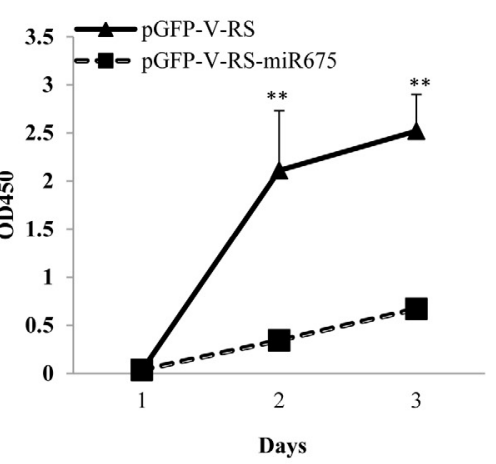

3

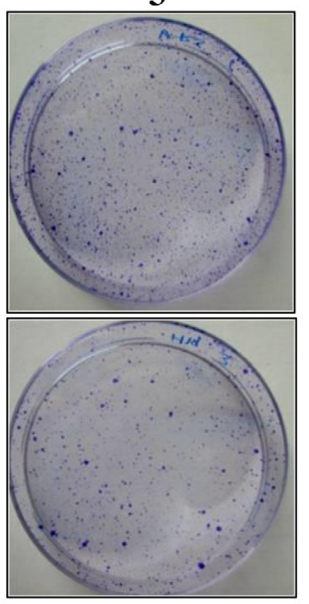

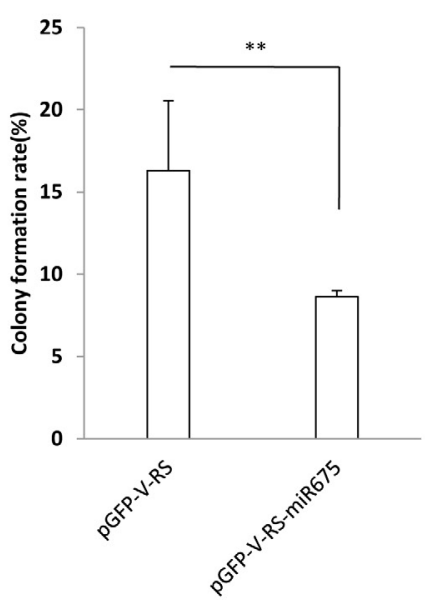




\section{C}

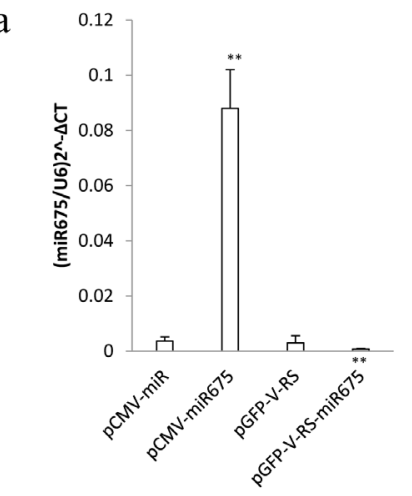

b

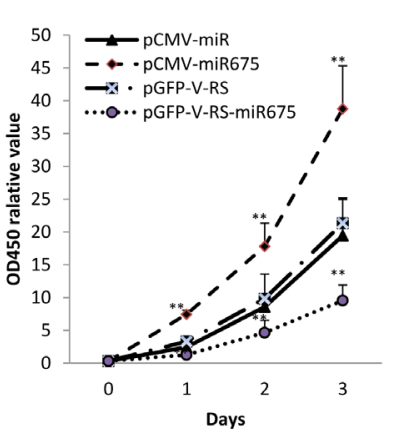

$\mathrm{d}$

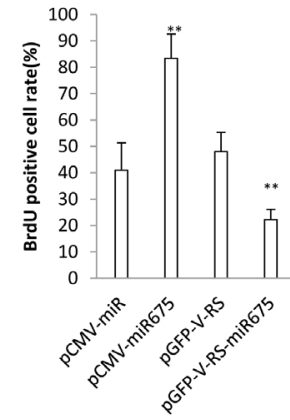

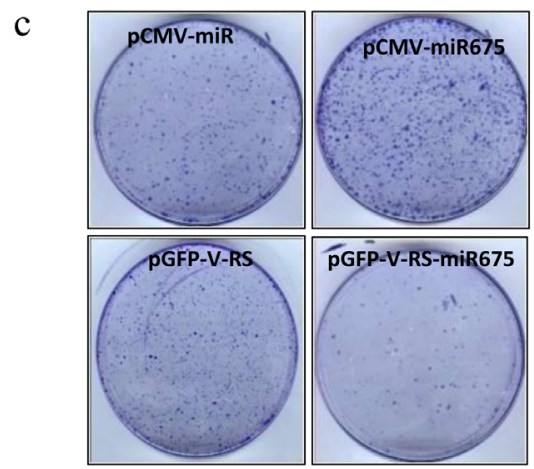

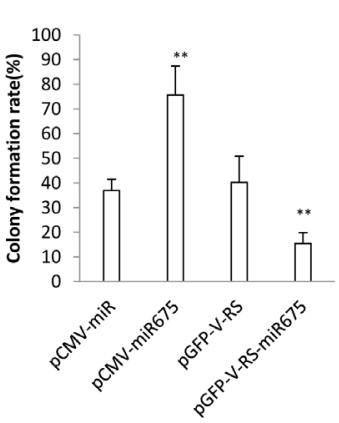

Figure 1: miR675 promotes liver cancer cells proliferation A. a. (left) The photography of the Hep3B cell lines transfected with pCMV-mir or pCMV-miR675. (right)Real-time RT-PCR for mature miR675 in miR675 overexpressed or mock control Hep3B stable cell lines (3\# and 6\# clone) ;U6 as internal control ;Data are means of value from three independent experiment, bar \pm SEM. **, $P<0.01 ; *$, $P<0.05$. b. Cell proliferation assay was performed in 96 -well format using the CCK 8 cells proliferation kit to determine the cell viability as described by the manufacturer. Each sample was assayed in triplicates for 3 days consecutively .Cell growth curve was based on the corresponding the relative values of OD450 and each point represents the mean of three independent samples. Data are means of value from three independent experiments, bar \pm SEM. **, $P<0.01 ; *, P<0.05$.c. (right)Cell plate colony formation ability assay. Data are means of value from three independent experiment, bar \pm SEM. **, $P<0.01 ; *, P<0.05$. (left)The photography of colonies from the cell lines indicated in left. B. a. (Left)Real-time RT-PCR for mature miR675 in miR675 knocked down or mock control Hep3B stable cell lines; 66 as internal control; Data are means of value from three independent experiment, bar \pm SEM. $* *, P<0.01$ ). (right) The photography of the cell lines transfected with pGFP-V-RS or pGFP-V-RS-miR675.b.Cell proliferation assay was performed in 96-well format using the CCK8 cells proliferation kit to determine the cell viability as described by the manufacturer. Each sample was assayed in triplicates for 3 days consecutively.Cell growth curve was based on the corresponding values of OD450 and each point represents the mean of three independent samples. Data are means of value from three independent experiments, bar \pm SEM. ${ }^{* *}, P<0.01$;,$P<0.05$.c. $($ right) Cell plate colony formation ability assay. Data are means of value from three independent experiment, bar \pm SEM. **, $P<0.01 ;^{*}, P<0.05$. $($ left)The photography of colonies from the cell lines indicated in left. C. a. Real-time RT-PCR for mature miR675 in miR675 overexpressed or knocked-down HepG2 stable cell lines; U6 as internal control ;Data are means of value from three independent experiment, bar \pm SEM. **, $P<0.01 ; *, P<0.05$. b. Cell proliferation assay was performed in 96 -well format using the CCK8 cells proliferation kit to determine the cell viability as described by the manufacturer. Each sample was assayed in triplicates for 3 days consecutively .Cell growth curve was based on the corresponding the relative values of OD450 and each point represents the mean of three independent samples. Data are means of value from three independent experiments, bar \pm SEM. **, $P<0.01 ; *, P<0.05$.c. (left)Cell plate colony formation ability assay.Data are means of value from three independent experiment, bar \pm SEM. **, $P<0.01 ; *, P<0.05$. (right) The photography of colonies from the cell lines indicated in left . d. Cell BrdU assay.Data are means of value from three independent experiment, bar \pm SEM. **, $P<0.01 ; *, P<0.05$.

2A (a, b), when mature miR675 was overexpressed, the xenograft tumor were produced in three mice $(0.1$ gram, 0.2 gram, 0.5 gram, respectively), while there was no xenograft tumor in control group $(0.2667 \pm 0.2081$ gram vs 0 gram, $n=3, P=0.045376$ ) (Figure 2Ab, left). The tumor formation ability in miR675 overexpressed group is significant higher than in control group (50\% vs $0 \%, n=6, P=0.00617$ ) (Figure $2 \mathrm{Ab}$, right), On the other hand, xenograft tumor was not produced in either mature miR675 knocked-down or RNAi control group. Pathological picture (hematoxylin-eosin staining) of three xenograft tumors from mature miR675 overexpressed group showed these tissue possessed poor-differentiation cells (5\# \& 6\# xenograft tumors) or less moderately cancer cells (4\# xenograft tumors) (Figure 2Ac), and stronger Proliferating cell nuclear antigen (PCNA) positive staining in 6\# xenograft (Figure 2Ad). There were 6 cases of $\mathrm{Balb} / \mathrm{C}$ nude mice in second animal test in vivo. for each 


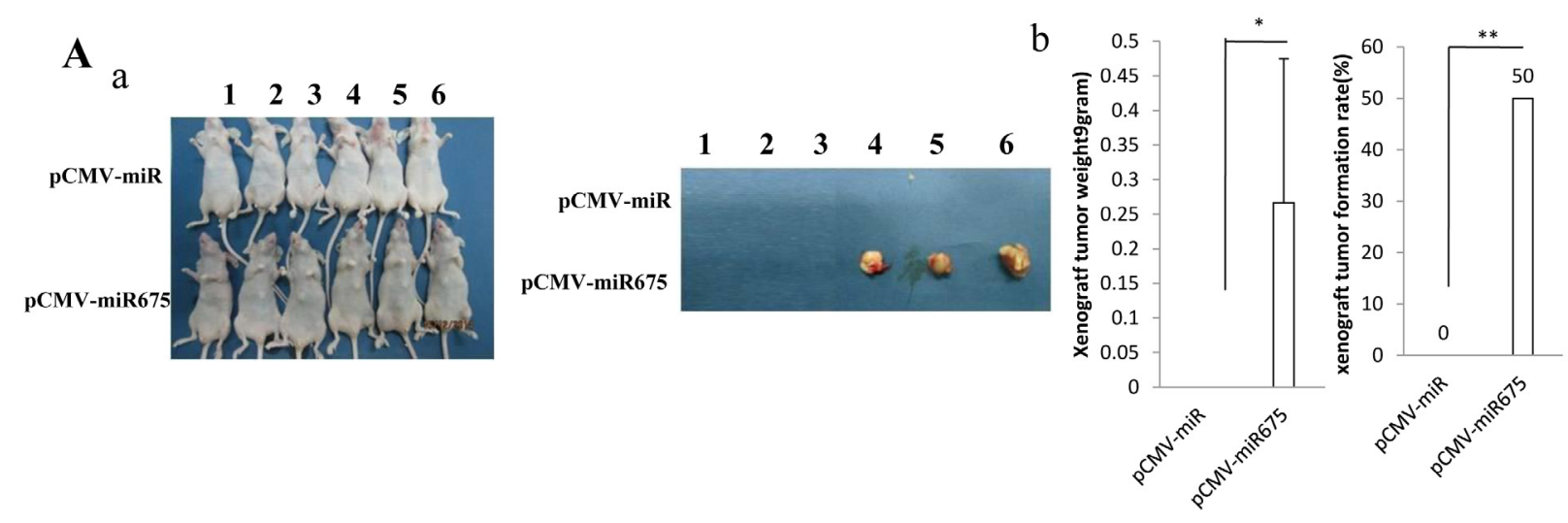

c
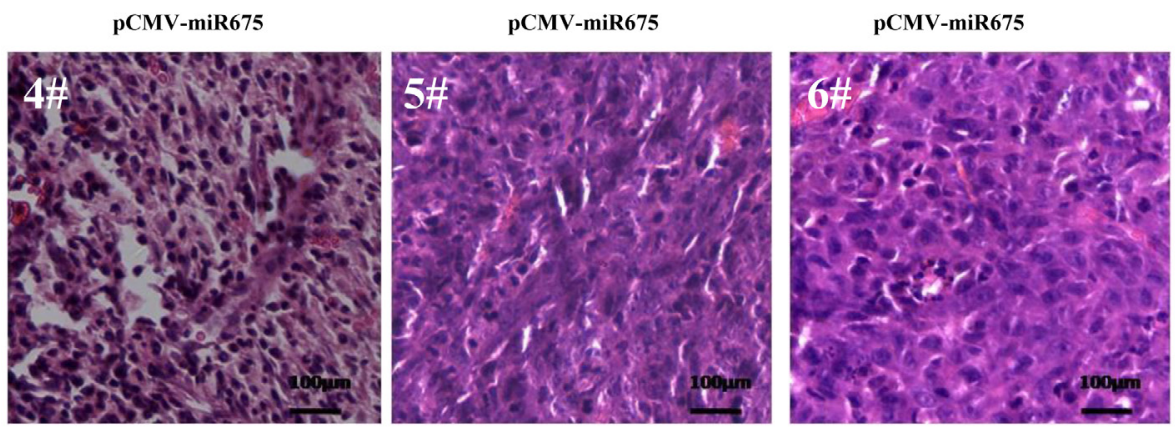

d

PCNA

B

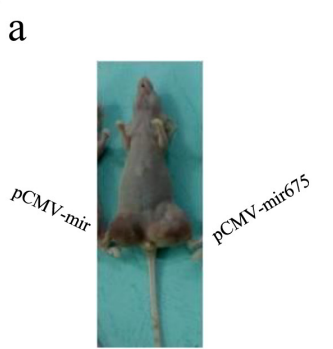

pCMV-miR

pCMV-miR675
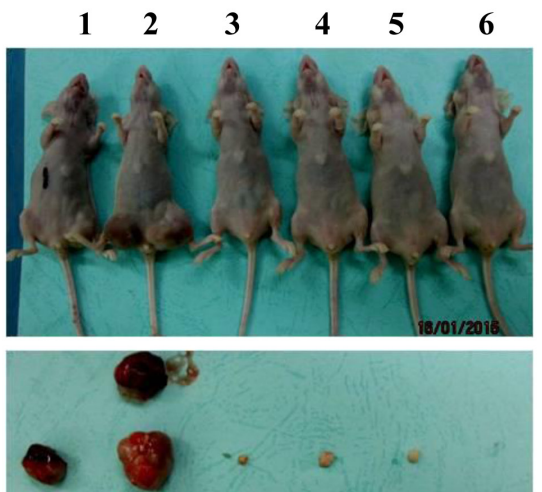

c

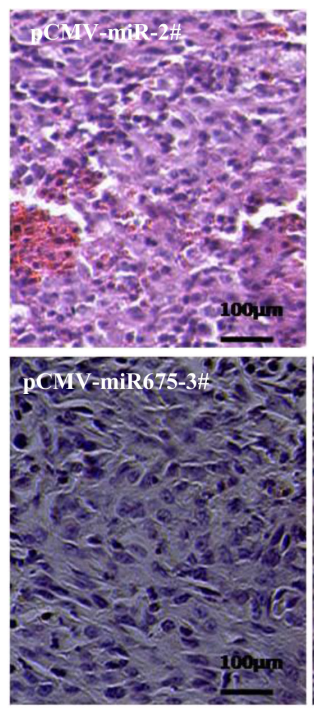

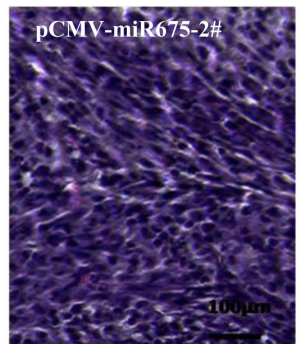

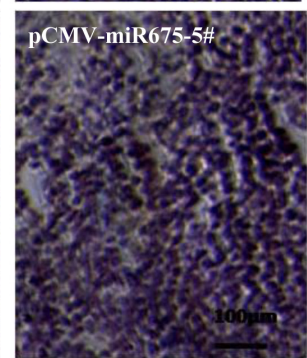

b
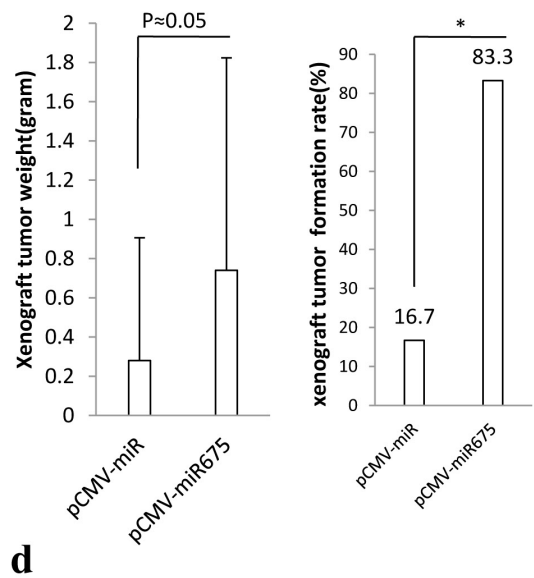

pCMV-miR-2\#

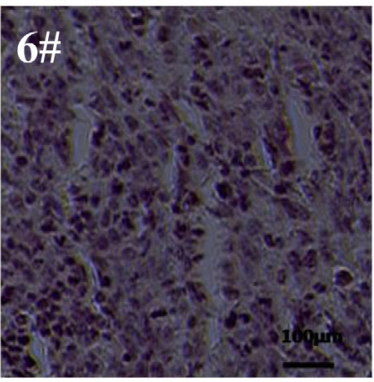

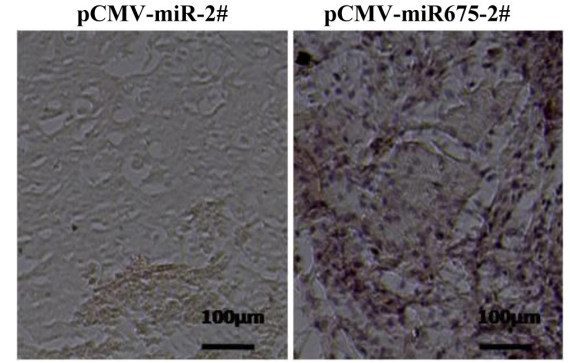


C

a

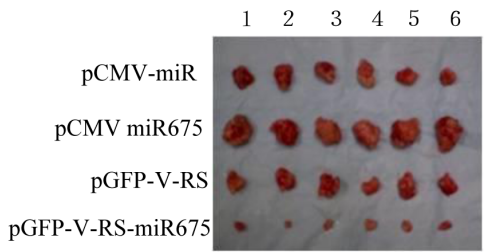

d
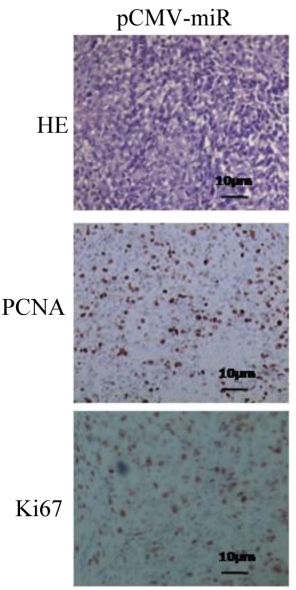

$\mathrm{b}$

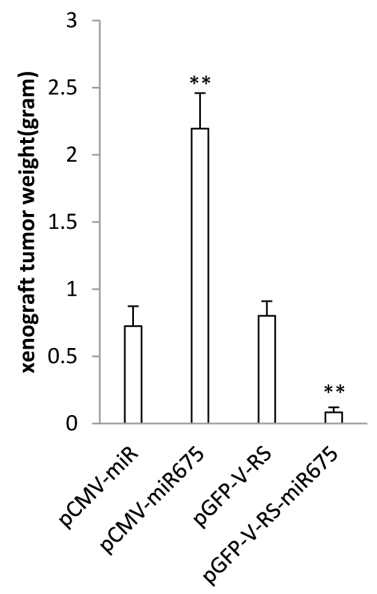

$\mathrm{C}$

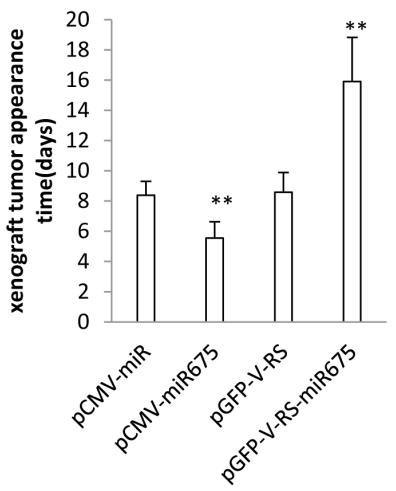

e
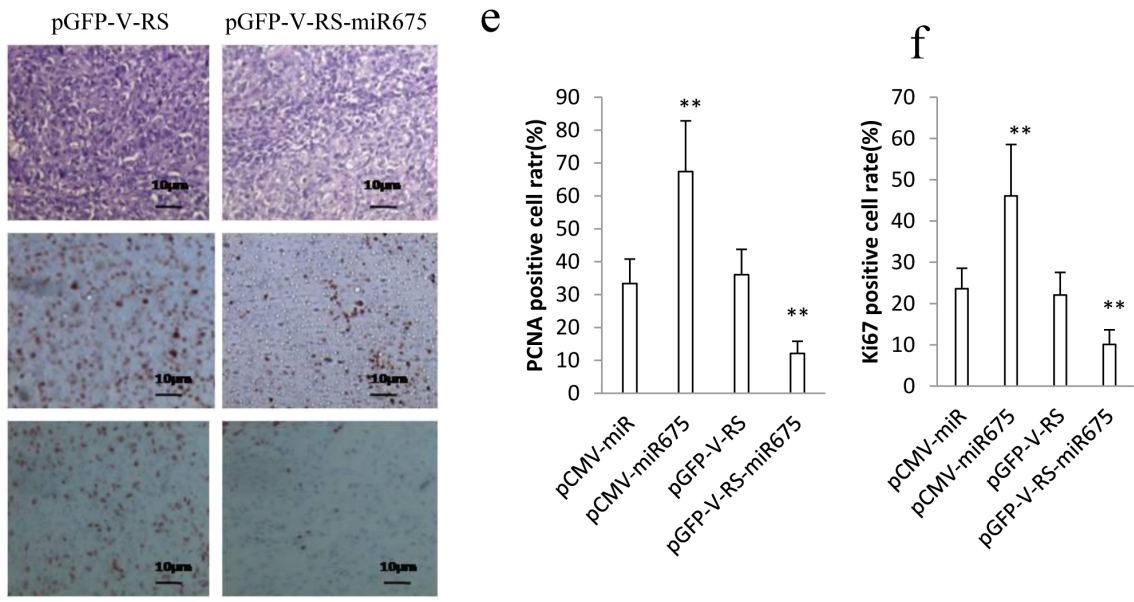

Figure 2: miR675 accelerates liver cancer cell growth in vivo A. a. The photography of xenograft tumors from Balb/C null mouse injected with Hep3B cells transfected with pCMV-miR or pCMV-miR675 subcutaneously at armpit.b. (left)The xenograft tumors formation ability (\%) in two groups indicated in lower. (right)The xenograft tumors weight (gram) in two groups indicated in lower. Data were means of value from six Balb/c mice, mean \pm SEM, $n=6,{ }^{*}, P<0.05 ; * *, P<0.01$. c. A portion of each xenograft tumor was fixed in $4 \%$ formaldehyde and embedded in paraffin, and the micrometers of sections $(4 \mu \mathrm{m})$ were made for hematoxylin-eosin (HE) staining (original magnification $\times 100$ ). d.anti-PCNA immunostainning in xenograft tumor sample (6\#). B. a. The photography of xenograft tumors from Balb/C null mouse injected with Hep3B cells transfected with pCMV-miR or pCMV-miR675 subcutaneously at armpit .b. The xenograft tumors weight ( $\mathrm{gram})$ in two groups indicated in left. Data were means of value from six Balb/c mice, mean $\pm \mathrm{SEM}, n=6$, *, $P<0.05$;**, $P<0.01$. c. A portion of each xenograft tumor was fixed in $4 \%$ formaldehyde and embedded in paraffin, and the micrometers of sections $(4 \mu \mathrm{m})$ were made for hematoxylin-eosin (HE) staining (original magnification $\times 100)$. $d$. anti-PCNA immunostainning in xenograft tumor sample (pCMV-miR 2\# and pCMV-miR675 2\#). C. a. The photography of xenograft tumors from Balb/C null mouse injected with HepG2 cells transfected with pCMV-miR, pCMV-miR675, pGFP-V-RS or pGFP-V-RS-miR675subcutaneously at armpit .b. The xenograft tumors weight (gram) in the four groups indicated in left. Data were means of value from six Balb/c mice, mean $\pm \mathrm{SEM}, n=6,{ }^{*}, P<0.05$;**, $P<$ 0.01. c. A portion of each xenograft tumor was fixed in $4 \%$ formaldehyde and embedded in paraffin, and the micrometers of sections $(4 \mu \mathrm{m})$ were made for hematoxylin-eosin (HE) staining (original magnification $\times 100$ ). $d$. anti-PCNA and anti-k67 immunostainning in xenograft tumor samples.

Balb/C mouse, miR675 overexpressed Hep3B cells were injected into right lower armpit area, miR control Hep3B cells were injected into left lower armpit area, miR675 knocked-down Hep3B cells were injected into right upper armpit area, RNAi control Hep3B cells were injected into left upper armpit area.As shown in Figure 2B (a, b), when mature miR675 was overexpressed, the xenograft tumors were produced in five mice (2.6gram, 0.8gram, 0.1 gram, 0.1 gram, 0.1 gram respectively), while there was one xenograft tumor in control group (1.4gram) $(0.28 \pm 0.62$ gram vs $0.74 \pm 1.08$ gram, $n=5, P \approx 0.05$ ) (Figure $2 \mathrm{Bb}$, left).The tumor formation ability in miR675 overexpressed group is significant higher than in control group $(83.3 \% v s$ $16.7 \%, n=6, P=0.0374$ ) Figure 2Bb, right). On the other hand, xenograft tumor was not produced in either mature miR675 knocked-down or control group. Pathological picture (hematoxylin-eosin staining) of xenograft tumors from mature miR675 overexpressed group showed these tissue possessed poor-differentiation cells (1\#, 2\#, 4\# xenograft tumors) or less moderately cancer cells ( $3 \#$ \& $5 \#$ 
xenograft tumors), and pathological picture of xenograft tumor from control group showed these tissue possessed well-differentiation cells (control $1 \#$ xenograft tumor) (Figure 2Bc), and stronger Proliferating cell nuclear antigen (PCNA) positive staining in 2\# miR675 xenograft tumor compared to $2 \#$ control xenograft tumor (Figure 2Bd).

As for HepG2 cell lines, there were $24 \mathrm{Balb} / \mathrm{C}$ nude mice in the third animal test in vivo. As shown in Figure 2Ca, when mature miR675 was overexpressed, the xenograft tumor weight increased approximately three folds when compared to the corresponding control group $(2.195 \pm 0.265$ grams versus $0.725 \pm 0.148$ grams, $P=0.000018)$. On the other hand, when mir675 was knocked down, the average xenograft tumor weight decreased to approximately one tenth of the control weight $(0.083 \pm 0.036$ grams versus $0.802 \pm 0.108$ grams, $P=$ 0.0000047) (Figure 2Cb).Mature miR675 overexpression resulted in early xenograft tumor formation compared to the control group $(5.55 \pm 1.08$ days versus $8.38 \pm 0.92$ days, $P=0.0050966)$. In contrast, the time of xenograft tumor appearance was prolonged in the mir675 knockdown group compared to the control group (15.91 \pm 2.92 days versus $8.58 \pm 1.31$ days, $P=0.001512$ ) (Figure $2 \mathrm{Cc})$. The xenograft tumor showed that tumor tissue possessed more poor-differentiation cells in mature mir675 overexpression group than that of control group , and less poor-differentiation cells in mir675 knockdown group than that of control group (Figure 2Cd upper).The PCNA-positive cells was significantly higher in miR675 overexpressed tumors compared to the vector control (67.42 $\pm 15.37 \%$ versus $33.33 \pm 7.47 \%, P=0.0023)$. Conversely, the percentage of PCNA positive cells was significantly lower in miR675 knockdown tumors (12.08 $\pm 3.74 \%$ versus 36.05 $\pm 7.69 \%, \quad P=0.000704)$ (Figure 2Cd middle and 2Ce). The Ki67-positive cells was significantly higher in miR675 overexpressed tumors compared to the vector control $(46.07 \pm 12.4 \%$ versus $23.58 \pm 4.99 \%, P=0.003784)$. Conversely, the percentage of Ki67 positive cells was significantly lower in miR675 knockdown tumors $(10.11 \pm 3.51 \%$ versus $22.56 \pm 5.49 \%, P$ $=0.000959$ ) (Figure 2Cd lower and 2Cf). Taken together, these observations demonstrate that miR675 accelerates liver cancer growth in vivo.

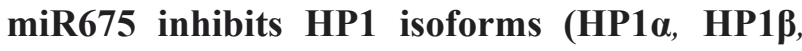 HP1 $\gamma$ )expression}

To address whether miR675 targets for $\mathrm{HP} 1 \alpha, \beta$, $\gamma$ and influences on their expression, we first performs the informatics analysis using MirTarget scanning soft (predicts biological targets of miRNAs by searching for the presence of conserved 8 mer and 7 mer sites that match the seed region of each miRNA)and BLAST analysis. As shown in Figure 3A, mature miR675 can match 3' untranslational region on HP1 $\alpha$ mRNA (BC006821) via eleven seed sequence (a); mature miR675 can match 3' untranslational region on HP1 $\beta$ mRNA (BC002609) via nine seed sequence (b); mature miR675 can match 3' untranslational region on HP1 $\gamma$ mRNA (AB030905) via ten seed sequence (c).Next, we constructed the luciferase report vector consisting of HP $1 \alpha / \beta / \gamma$ 3'UTR containing miR675 targeting site and their corresponding mutant plasmid of HP1 $\alpha / \beta / \gamma$ 3'UTR, and pCMV-miR mutant miR675.As shown in Figure 3B, the HP1 $23^{\prime}$ UTR luciferase activity was significantly reduced in mature miR675 overexpressed Hep3B cells compared to control group $(p<0.01)$ and increased in mature miR675 knocked down Hep3B cells compared to control group $(p<0.01)$. Nevertheless, the HP1 $\alpha 3$ 'UTR luciferase activity was significantly not altered in Hep3B cell lines transfected with pCMV-mutant miR675 plus pMirtarget-HP1 $\alpha 3$ 'UTR, pMirtarget-mutant HP1 $\alpha 3^{\prime}$ UTR or pCMV-miR675 plus pMirtarget-mutant HP1 $\alpha 3^{\prime}$ UTR $(P>0.05)$. The HP1 $\beta$ 3'UTR luciferase activity was significantly reduced in mature miR675 overexpressed Hep3B cells compared to control group $(p<0.01)$ and increased in mature miR675 knocked down Hep3B cells compared to control group $(p<0.01)$.Nevertheless, the HP1 $133^{\prime}$ UTR luciferase activity was significantly not altered in Hep3B cell lines transfected with pCMV- mutant miR675 plus pMirtargetHP1 $\beta 3$ 'UTR, pMirtarget-mutant HP1 $\beta 3$ 'UTR or pCMVmiR675 plus pMirtarget-mutant HP1 $\beta 3$ 'UTR $(P>0.05)$. The HP1 $\gamma 3$ 'UTR luciferase activity was significantly reduced in mature miR675 overexpressed Hep3B cells compared to control group $(p<0.01)$ and increased in mature miR675 knocked down Hep3B cells compared to control group $(p<0.01)$.Nevertheless, the HP1 $\gamma 3$ 'UTR luciferase activity was significantly not altered in Hep3B cell lines transfected with pCMV- mutant miR675 plus pMirtarget-HP1 $\gamma 3$ 'UTR, pMirtarget-mutant HP1 $\gamma 3$ 'UTR or pCMV- miR675 plus pMirtarget-mutant HP1 $\gamma 3$ 'UTR $(P>0.05)$. Then we performed the western blotting using anti-HP1 $\alpha$, anti-HP1 $\beta$ and anti-HP1 $\gamma$.As shown in Figure $3 \mathrm{C}, \mathrm{HP} 1 \alpha, \mathrm{HP} 1 \beta, \mathrm{HP} 1 \gamma$ expression were significantly decreased in mature miR675 overexpressed Hep3B cells compared to control group (pCMV-miR). HP1 $\alpha$, HP1 $\beta$, HP $1 \gamma$ expression were significantly increased in miR675 knockdown Hep3B cells compared to control group (pGFP-V-RS) (Figure 3D).Moreover, our findings showed that mature miR675 overexpression reduced the expression of Cadherin 13 , RUNX1 and RB1 in Hep3B cell lines (Figure 3E). Collectively, our findings suggest miR675 targets for HP1 isoforms including HP1 $\alpha$, HP1 $\beta$, HP1 $\gamma$ and inhibits their expression in liver cancer cells. 
Aa

mutant has-miR675 5' 5' -TGGTGCGGAGAGTGTTAACAAGG-3' has-miR675 5' -TGGTGCGGAGAGGGCCCACAGTG-3' | | | | || | ||||

Position of HP1 $\alpha 3^{\prime}$ UTR 3'-AAAA A A AAAACACCTCGTG TCAC-5'

mutantHP1 $\alpha 3^{\prime}$ UTR 5'AAAA A A AAAACACCTCAGAACAC-5'

C mutant has-miR675 5' -TGGTGCGGAGAG TGTTAACAGT G-3'

has-miR675 5' -TGGTGCGGAGAGGGCC CACAGTG-3'

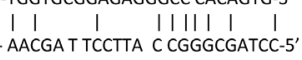

Position of HP1Y3' UTR 3'- AACGA T TCCTTA C CGGGCGATCC-5

B a

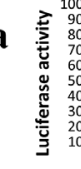

mutantHP1Y3' UTR
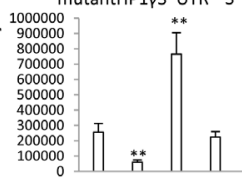

c

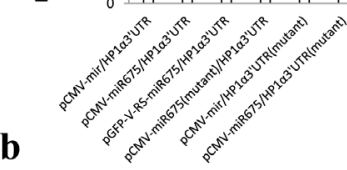

b

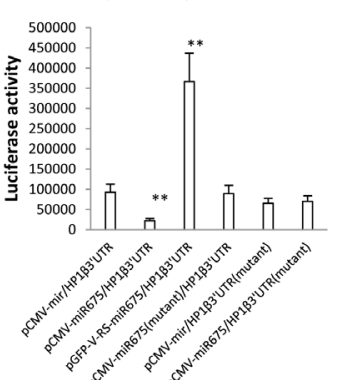

D
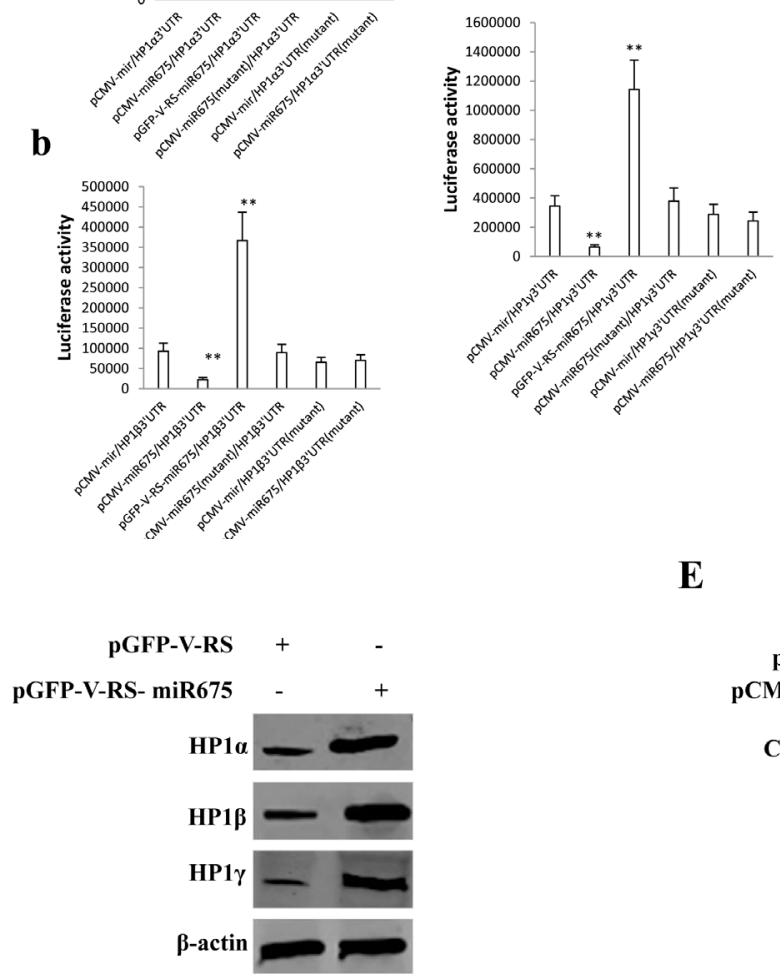

b

mutant has-miR675 5' -TGGTGCGGAGAGGGTTA GCGGTG-3'

has-miR675 5' -TGGTGCGGAGAGGGCCCA CAGTG-3'

Position of HP1B3' UTR $3^{\prime}$-GAAGGGTGAACTTTGGGTGT CAG-5

mutantHP1 $133^{\prime}$ UTR $3^{\prime}$-GAA GGGTGAACTTTCCCTGT GGG-5’

C

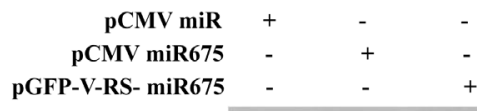

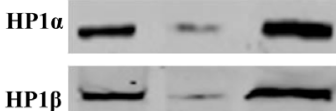
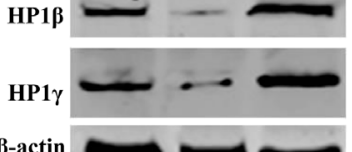

$\beta$-actin

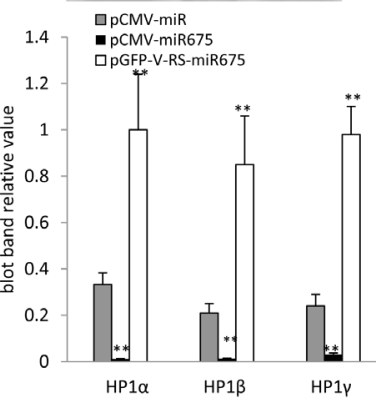

$\mathbf{E}$
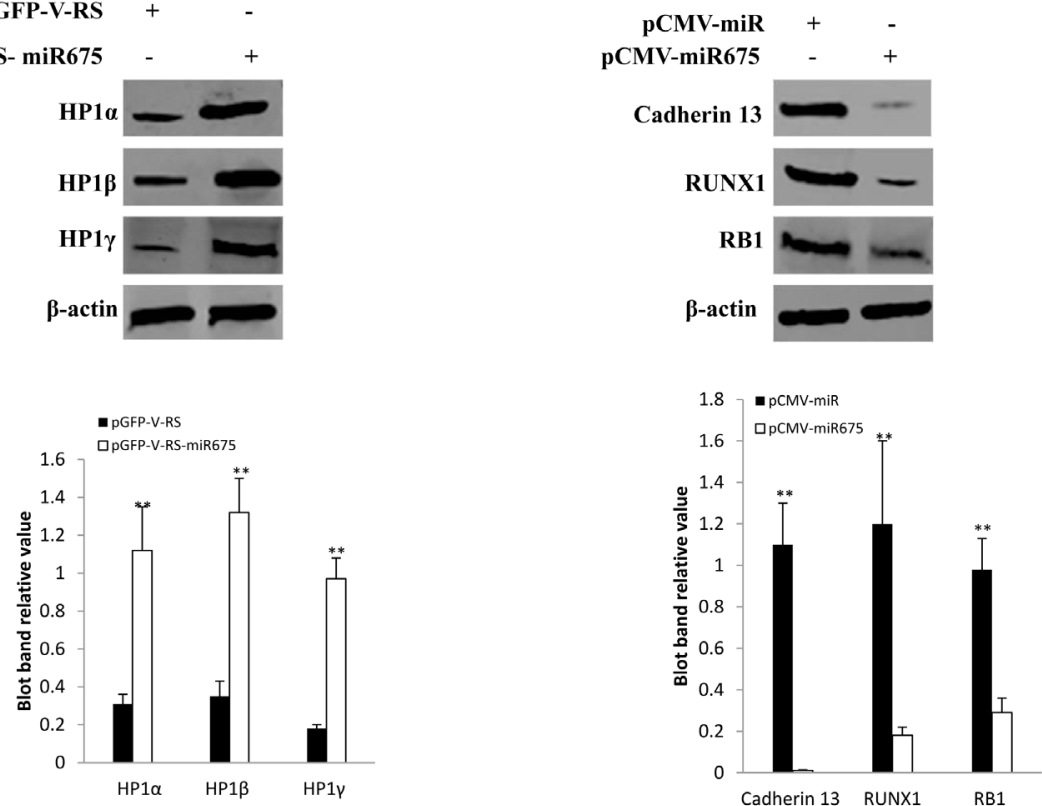

Figure 3: miR675 inhibits HP1 $\alpha$, HP1 $\beta$, HP1 $\gamma$ expression in human liver cancer cells. A. MirTarget bioinformatics analysis. a. Mir675 targets for human HP1 $\alpha$ 3'UTR ; b.Mir675 targets for huamn HP1 $\beta$ 3'UTR;c.Mir675 targets for HP1 $\gamma$ 3'UTR. B. HP1 $\alpha, \beta$, $\gamma$-3'-UTR-Luciferase activity assay. Hep3B cells were transfected with pMirTarget-control, pMirTarget-HP1 $\alpha 3$ 'UTR, pMirTarget-mutant HP1 $\alpha 3^{\prime}$ UTR, pMirTarget-HP1 $\beta 3$ 'UTR, pMirTarget-mutant HP1 $\beta 3$ 'UTR, pMirTarget-HP1 $\gamma 3^{\prime}$ 'UTR, pMirTarget-mutant HP1 $\gamma 3^{\prime}$ 'UTR, pCMV-miR, pCMV-miR675, pCMV-mutant miR675 respectively. Data are means of value from three independent experiment, bar \pm SEM. **, $P<0.01$. C. Western blotting analysis using anti-HP1 $\alpha$, anti-HP1 $\beta$, anti-HP $1 \gamma$ in Hep3B cell lines transfected with pCMV-miR , pCMV-miR675, pGFP-V-RS-miR675, respectively. $\beta$-actin as internal control. D. Western blotting analysis using anti-HP1 $\alpha$, anti-HP1 $\beta$, anti-HP1 $\gamma$ in Hep3B cell lines transfected with pGFP-V-RS , pGFP-V-RS-miR675, respectively. $\beta$-actin as internal control. C.Western blotting analysis using anti-HP1 $\alpha$, anti-HP1 $\beta$, anti-HP1 $\gamma$ in Hep3B cell lines transfected with pCMV-miR, pCMV-miR675, pGFP-V-RSmiR675, respectively. $\beta$-actin as internal control. D.Western blotting analysis using anti-Cadherin 13, anti-RUNX1, anti-RB1 in Hep3B cell lines transfected with pCMV-miR, pCMV-miR675, respectively. $\beta$-actin as internal control. 


\section{miR675 induces EGR1 expression through reducing HP1a}

We infer miR675 may alter early growth response protein1 (EGR1) expersion epigenetically. To explore whether mature miR675 alters EGR1 expression, we consider to reveal whether mature miR675 impacts on the Histone 3 modification in liver cancer cells. At the first time, we performed the Co-immunoprecipitation (Co-IP) to analyse the interaction between SUV39h1 and HP1 $\alpha$, SUV39h1 and Histone3, SUV39H1 and H3K27Ac, EZH2 and SUZ12 in Hep3B cells transfected with pCMV-miR, pCMV-miR675, pCMV-miR675 plus pcDNA3.1-HP1 $\alpha$. Our results showed that the interplay between SUV39H1 and $\mathrm{HP} 1 \alpha$, SUV39H1 and Histone 3 was attenuated in mature miR675 overexpressed Hep3B compared to control group (Figure 4Aa) and that the interplay between SUV39H1 and HP1 $\alpha$, SUV39H1 and Histone3 was increased in mature miR675 knockdown Hep3B compared to control group (Figure 4Ab). Obviously, this is due to a reduction of HP1 expression in miR675 overexpressed Hep3B. As shown in Figure 4Ba, the interplay between SUV39H1 and H3K27Ac, H3K27Ac and HP1 $\alpha$ were weakened in mature miR675 overexpressed Hep3B compared to control group. However, the decreased interaction between SUV39H1 and H3K27Ac was rescued and interaction between H3K27Ac and HP1 $\alpha$ was slightly increased in Hep3B cells transfected with pCMV-miR675 plus pcDNA3.1-HP1 $\alpha$ compared to the control. The interplay between SUV39H1 and H3K27Ac, H3K27Ac and HP1 $\alpha$ were enhanced in mature miR675 knockdown Hep3B compared to control group (Figure $4 \mathrm{Bb}$ ).In addition, the interplay between EZH2 and SUZ12, SUZ12 and HP1 $\alpha$ were attenuated in mature miR675 overexpressed Hep3B compared to control group. However, this interaction between EZH2 and SUZ12 was not altered and this interaction between SUZ12 and HP1 $\alpha$ was increased in Hep3B cells transfected with pCMV-miR675 plus pcDNA3.1-HP1 $\alpha$ compared to the control (Figure 4Ca). The interplay between EZH2 and SUZ12, SUZ12 and HP1 $\alpha$ were increased in mature miR675 knockdown Hep3B compared to control group (Figure $4 \mathrm{Cb}$ ). Further on, we detected the Histone3 modification (H3K9me3, H3K27me3, H3K27Ac, pHistone 3 and H3K4me3) and PKM2 expression in liver cancer. In Hep3B cells, mature miR675 was significantly overexpressed in Hep3B cell lines transfected with pCMV-miR675 or pCMV-miR675 plus pcDNA3.1-HP1 $\alpha$ compared to control (Figure 4Da).HP1 $\alpha$ expression was significantly reduced in Hep3B cell line transfected with pCMV-miR675 and significantly overexpressed in Hep3B cell line transfected with pCMV-miR675 plus pcDNA3.1HP1 $\alpha$ compared to control (Figure 4Db, the first row from upper to lower). Both $\mathrm{H} 3 \mathrm{~K} 9 \mathrm{me} 3$ and $\mathrm{H} 3 \mathrm{~K} 27 \mathrm{me} 3$ were significantly decreased in Hep3B cell line transfected with pCMV-miR675.Notably, both H3K9me3 and H3K27me3 were slightly increased in Hep3B cell line transfected with pCMV-miR675 plus pcDNA3.1-HP1 $\alpha$ compared to control (Figure 4Db, the second \& third row from upper to lower). H3K27Ac, pHistone 3 and $\mathrm{H} 3 \mathrm{~K} 4 \mathrm{me} 3$ were significantly increased in Hep3B cell line transfected with pCMV-miR675. It was worth noting that H3K27Ac and $\mathrm{H} 3 \mathrm{k} 4 \mathrm{me} 3$ was slightly decreased and pHistone was not altered in Hep3B cell line transfected with pCMV-miR675 plus pcDNA3.1-HP1 $\alpha$ compared to control (Figure $4 \mathrm{Db}$, the forth , fifth, sixth row from upper to lower). Strikingly, mono PKM2 (58KD) , PKM2 dimer (116KD) and PKM2 tertamer $(232 \mathrm{KD})$ were significantly increased in Hep3B cell line transfected with pCMV-miR675. However, mono PKM2 (58KD) , PKM2 dimer (116KD) and PKM2 tertamer (232KD) were significantly reduced in Hep3B cell line transfected with pCMV-miR675 plus pcDNA3.1HP1 $\alpha$ compared to control (Figure 4Dc). HP1 $\alpha$ expression was significantly increased in Hep3B cell line transfected with pGFP-V-RS-miR675 compared to control (Figure 4Dd, the first row from upper to lower). Both H3K9me3 and $\mathrm{H} 3 \mathrm{~K} 27 \mathrm{me} 3$ were significantly increased in Hep3B cell line transfected with pGFP-V-RS-miR675 compared to control (Figure 4Dd, the second \& third row from upper to lower). H3K27Ac, pHistone 3 and $\mathrm{H} 3 \mathrm{~K} 4 \mathrm{me} 3$ were significantly decreased in Hep3B cell line transfected with pGFP-V-RS-miR675 compared to control (Figure 4Dd, the forth, fifth, sixth row from upper to lower). Strikingly, mono PKM2 (58KD), PKM2 dimer (116KD) and PKM2 tertamer (232KD) were significantly decreased in Hep3B cell line transfected with pGFP-V-RS-miR675 compared to control (Figure 4De). Next, we performed the Chromatin Immunoprecipitation (CHIP) assay in liver cancer cell line. As shown in Figure 4E (a, b), the loading of H3Kme9, H3K27me3, HP1 $\alpha$ on the EGR1 promoter region were attenuated in Hep3B cell line transfected with pCMV-miR675.However, the loading of $\mathrm{H} 3 \mathrm{Kme} 9, \mathrm{H} 3 \mathrm{~K} 27 \mathrm{me} 3$, HP1 $\alpha$ on the EGR1 promoter region were enhanced in Hep3B cell line transfected with pCMV-miR675 plus pcDNA3.1-HP1 $\alpha$ compared to control. By contraries, the loading of H3K27Ac on the EGR1 promoter region were increased in Hep3B cell line transfected with pCMV-miR675 compared to control .It is noteworthy that the loading of H3K27Ac on the EGR1 promoter region were slightly weakened in Hep3B cell line transfected with pCMV-miR675 plus pcDNA3.1HP1 $\alpha$ compared to contro1. Then we performed the luciferase activity assay in aforementioned Hep3B.cell lines. As shown in Figure 4F, EGR1 promoter luciferase activity was significantly increased in Hep3B cell line transfected with pCMV-miR675 compared to control $(149207.3 \pm 32350.21$ vs $27669.1 \pm 5418.8, P<0.01)$. However, EGR1 promoter luciferase activity was slightly decreased in Hep3B cell line transfected with pCMVmiR675 plus pcDNA3.1-HP1 $\alpha$ compared to control (8549.0 \pm 2267.5 vs $27669.1 \pm 5418.8, P<0.01)$. Finally, we analyse the EGR1 expression by western blotting 
A

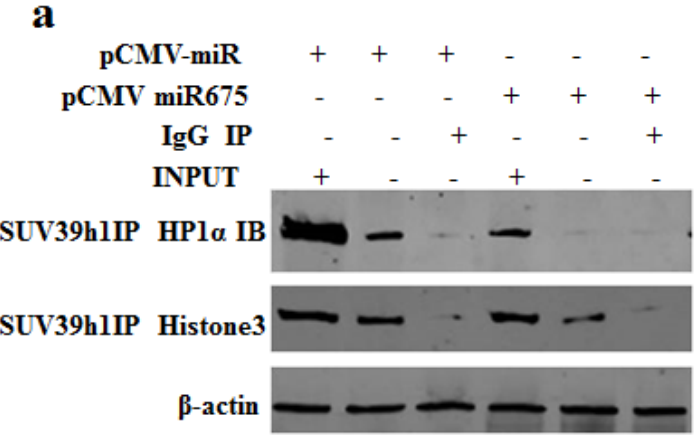

B

$\mathbf{a}$

$\begin{array}{rllllll}\text { pCMV-miR } & + & + & - & - & - & - \\ \text { pCMV-miR675 } & - & - & + & + & + & + \\ \text { HP1 } \alpha & - & - & - & - & + & + \\ \text { H3K27Ac IP } & + & - & + & - & + & - \\ \text { IgG IP } & - & + & - & + & - & + \\ \text { SUV39H1 } & & & & & \\ \text { HP1 } \alpha & & - & & & & \end{array}$

C a
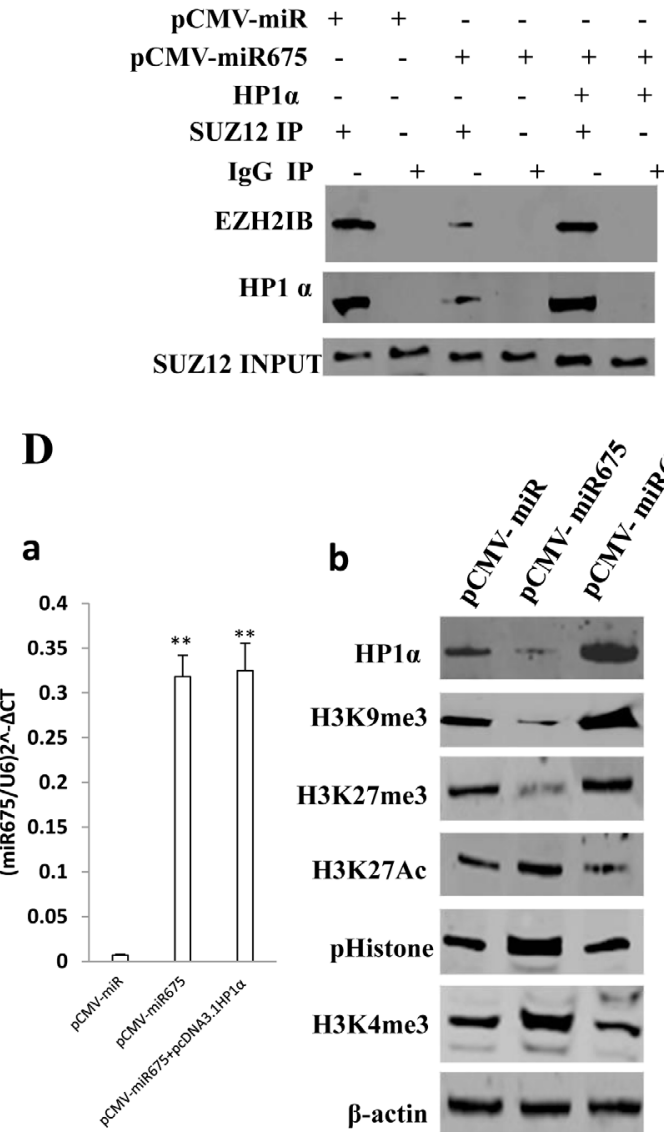

b

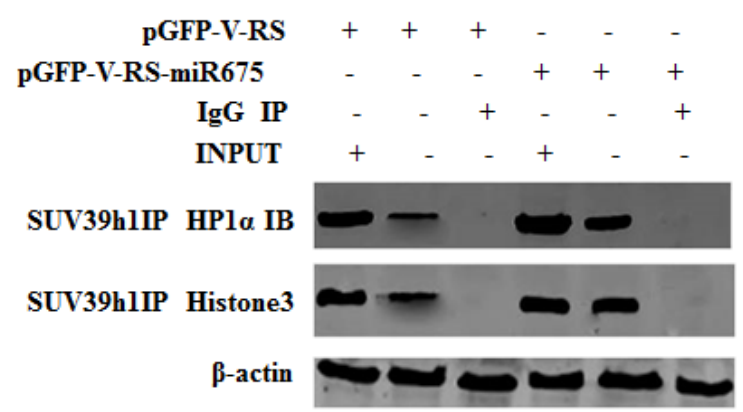

b

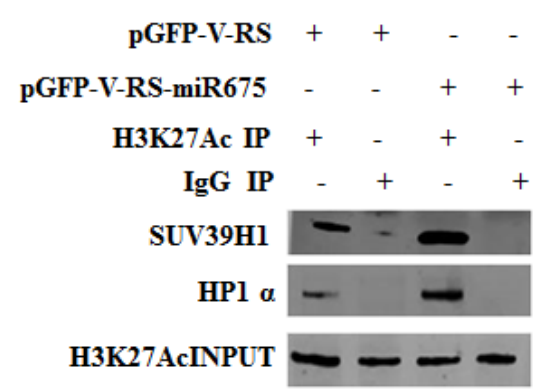

b

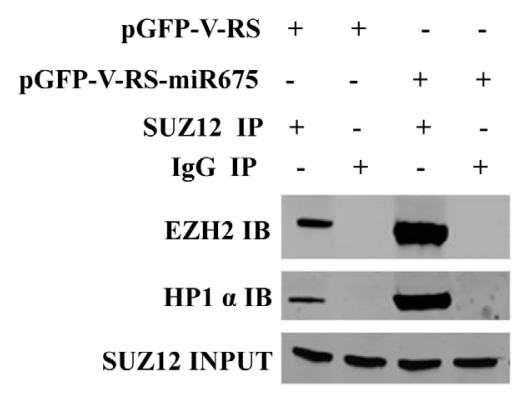

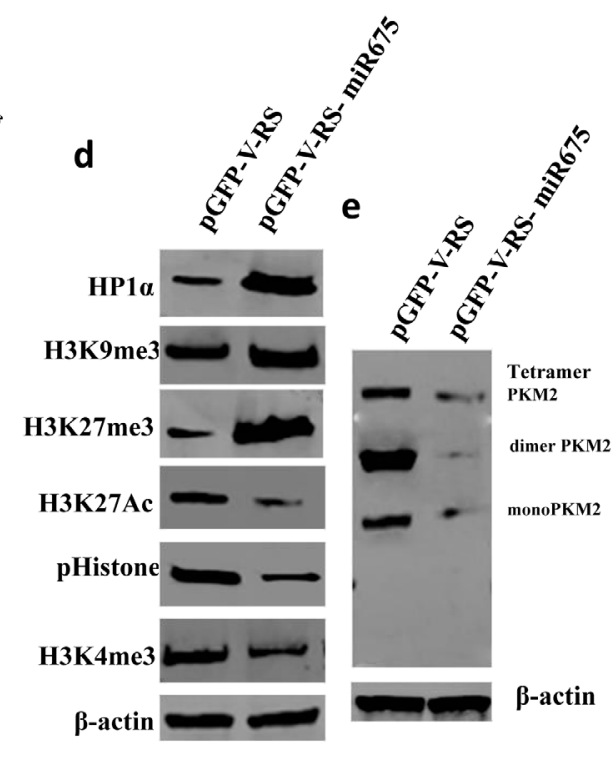




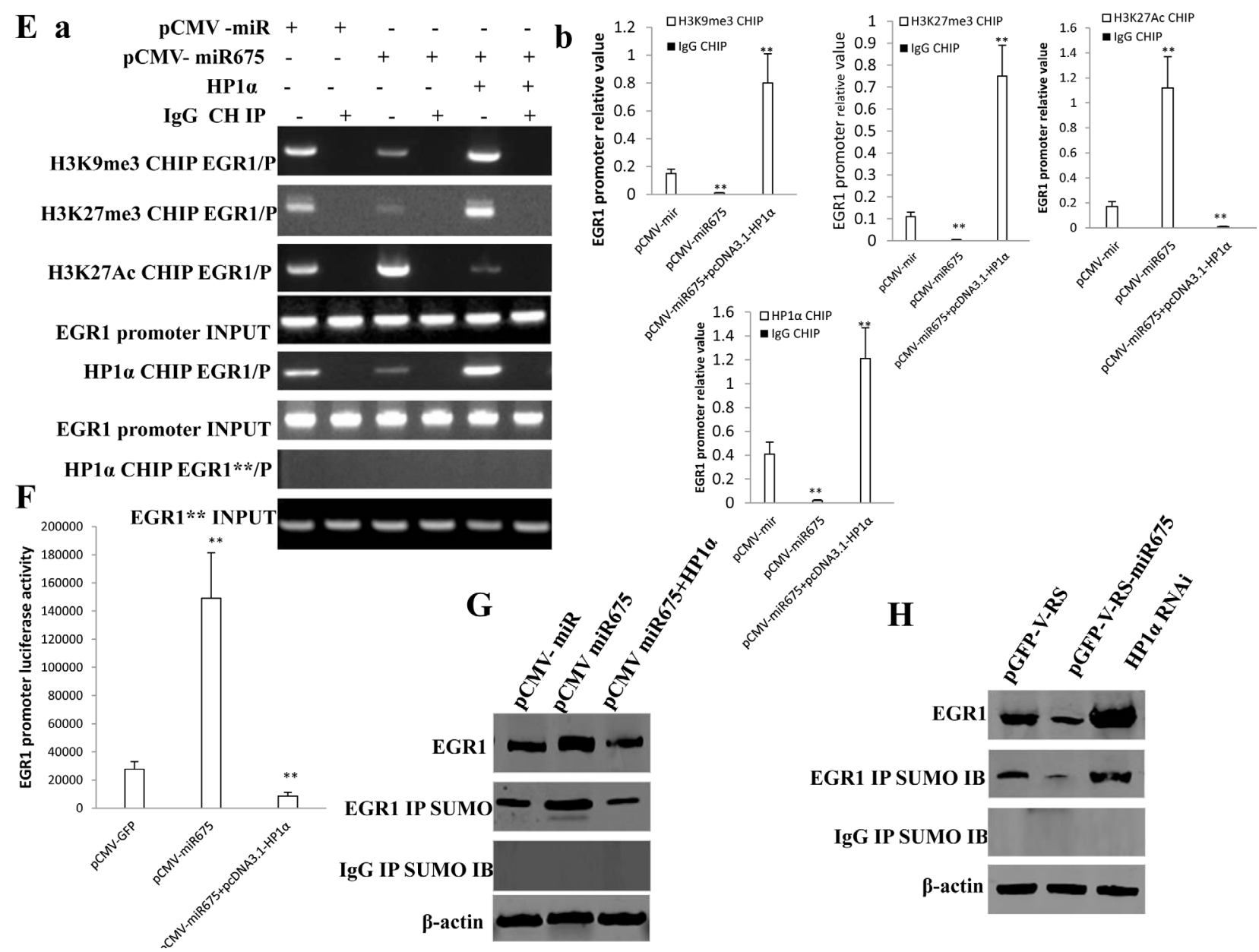

Figure 4: miR675 enhances EGR1 via reducing HP1 $\boldsymbol{\alpha}$ in human liver cancer cells. A. a.Co-Immunoprecipitation (IP) with anti-SUV39h1 followed by western blotting with anti-HP1 $\alpha$ or anti-Histone3 in miR675 overexpressied and control Hep3B. IgG IP as negative control. INPUT refers to western blotting with anti-HP1 $\alpha . \beta$-actin as internal control. b. Co-Immunoprecipitation (IP) with antiSUV39h1 followed by western blotting with anti-HP1 $\alpha$ or anti-Histone3 in miR675 knockdown and control Hep3B. IgG IP as negative control. INPUT refers to western blotting with anti-HP1 $\alpha$. $\beta$-actin as internal control. B. a.Co-Immunoprecipitation (IP) wih anti-H3k27Ac followed by western blotting with anti-SUV39H1 in miR675 overexpressied, miR675 plus HP1 $\alpha$ overexpressed and control Hep3B. IgG IP as negative control. INPUT refers to western blotting with anti-SUV39h1. b.Co-Immunoprecipitation (IP) wih anti-H3k27Ac followed by western blotting with anti-SUV39H1 in miR675 knockdown control Hep3B. IgG IP as negative control. INPUT refers to western blotting with anti-SUV39h1. C. a.Co-Immunoprecipitation (IP) with anti-SUZ12 followed by western blotting with anti-EZH2, anti-HP1 $\alpha$ in miR675 overexpressied, miR675 plus HP1 $\alpha$ overexpressed and control Hep3B. IgG IP as negative control. INPUT refers to western blotting with anti-EZH2. b. Co-Immunoprecipitation (IP) wih anti-SUZ12 followed by western blotting with anti-EZH2, anti-HP1 $\alpha$ in miR675 knockdown and control Hep3B. IgG IP as negative control. INPUT refers to western blotting with anti-EZH2. D. a. Real-time RTPCR for mature miR675 in Hep3B cells transfected with pCMV-miR, pCMV-miR675, pCMV-miR675 plus pcDNA3.1-HP1 $\alpha$ respectively. U6 as internal control. b. Western blotting with anti-HP1 $\alpha$, anti-H3K9me3, anti-H3K27me3, anti-pHistone3, H3K27Ac, anti-H3K4me3 in Hep3B cells transfected with pCMV-miR, pCMV-miR675, pCMV-miR675 plus pcDNA3-HP1 $\alpha$ respectively. $\beta$-actin as internal control. c.Western blotting with anti-PKM2 in Hep3B cells transfected with pCMV-miR, pCMV-miR675, pCMV-miR675 plus pcDNA3-HP1 $\alpha$ respectively. $\beta$-actin as internal control. d.Western blotting with anti-HP1 $\alpha$, anti-H3K9me3, anti-H3K27me3, anti-pHistone3, H3K27Ac, anti-H3K4me3 in Hep3B cells transfected with pGFP-V-RS, pGFP-V-RS-miR675 respectively. $\beta$-actin as internal control. e.Western blotting with anti-PKM2 in Hep3B cells transfected with pGFP-V-RS, pGFP-V-RS-miR675 respectively. $\beta$-actin as internal control. E. a.Chromatin Immunoprecipitation (CHIP) with anti-H3K9me3, anti-H3K27me3, anti-H3K27Ac and anti-HP1 $\alpha$ followed by PCR with EGR1 promoter primers in miR675 overexpressied, miR675 pluc HP1 $\alpha$ overexpressed and control Hep3B. IgG IP as negative control. IgG CHIP as negative control. EGR1 promoter DNA sequence as INPUT. EGR1 DNA** as negative control.b. Chromatin Immunoprecipitation (CHIP) with anti-H3K9me3, anti-H3K27me3, anti-H3K27Ac and anti-HP1 $\alpha$ followed by real-time PCR with EGR1 promoter primers in miR675 overexpressied, miR675 pluc HP1 $\alpha$ overexpressed and control Hep3B. F. EGR1 promoter luciferase activity assay in Hep3B cells transfected with pCMV-miR, pCMV-miR675, pCMV-miR675 plus pcDNA3-HP1 $\alpha$ respectively. Each value was presented as mean \pm standard error of the mean (SEM). G. Western blotting with anti-EGR1 and CO-IP with anti-SUM (EGR1 IB) in Hep3B cells transfected with pCMV-miR, pCMV-miR675, pCMV-miR675 plus pcDNA3-HP1 $\alpha$ respectively. $\beta$-actin as internal control. H. Western blotting with anti-EGR1 and CO-IP with anti-SUM (EGR1 IB) in Hep3B cells transfected with pGFP-V-RS, pGFP-V-RS-miR675, pGFPV-RS- HP1 $\alpha$ respectively. $\beta$-actin as internal control. 
and EGR1 sumoylation by Co-IP in above-mentioned Hep3B cell lines. As showed in Figure 4G, both EGR1 expression and SUM-EGR1 were significantly increased in Hep3B cell line transfected with pCMV-miR675 compared to control. However both EGR1 expression and SUM-EGR1 were slightly decreased in Hep3B cell line transfected with pCMV-miR675 plus pcDNA3.1-HP1 $\alpha$ compared to control. Significantly, both EGR1 expression and SUM-EGR1 were significantly decreased in Hep3B cell line transfected with pGFP-V-RS-miR675 compared to control. Moreover, both EGR1 expression and SUMEGR1 were significantly increased in Hep3B cell line transfected with pGFP-V-RS-HP1 $\alpha$ compared to control (Figure 4H). Taken together, these observations suggest that mature miR675 reduced the complex output of HP1 $\alpha$-SUV39H1-Histone3, HP1 $\alpha-S U V 39 H 1-H 3 K 27 A c$, HP1 $\alpha-S U Z 12-E Z H 2$ which caused the reduction of H3K9me3, H3K27me3 and increment of H3K27Ac, and increased EGR1 turnout through transcriptional regulation ultimately.

\section{miR675 upregulates H19 expression by increasing the EGR1 occupancy on H19 imprinting control region (ICR)}

To explore whether miR675 alters long noncoding RNA H19 expression through EGR1 action, we have a reason to consider whether EGR1 may control H19 transcriptional activity. Bioimformatics analysis suggests that EGR1 binding site (5'-CGCCCCCGC-3' and 5'-GCGGGGGCG-3') exists on the H19 promoter region (Figure 5A).Next, we performed the DNA pulldown assay using the EGR1 site probe. As shown in Figure 5B, The binding capacity of EGR1 to EGR1 site was significantly enhanced in Hep3B cell line transfected with pCMVmiR675 compared to control. However, The binding capacity of EGR1 to EGR1 site was slightly decreased in Hep3B cell line transfected with pCMV-miR675 plus pcDNA3.1-HP1 $\alpha$ compared to control to control. Next, we performed the Chromatin Immunoprecipitation (CHIP) assay in Hep3B liver cancer cell line. As shown in Figure 5C, the loading of EGR1 on the H19 promoter region was increased in Hep3B cell line transfected with pCMV-miR675.However, the loading of EGR1 on the H19 promoter region was slightly attenuated in Hep3B cell line transfected with pCMV-miR675 plus pcDNA3.1-

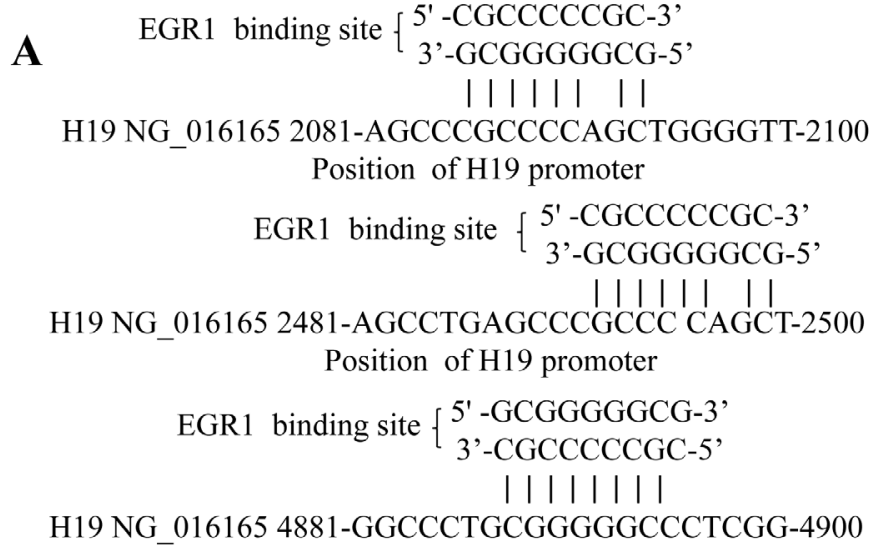

Position of $\mathrm{H} 19$ promoter

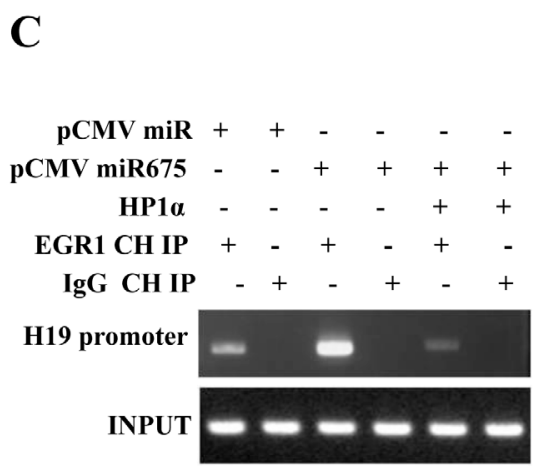

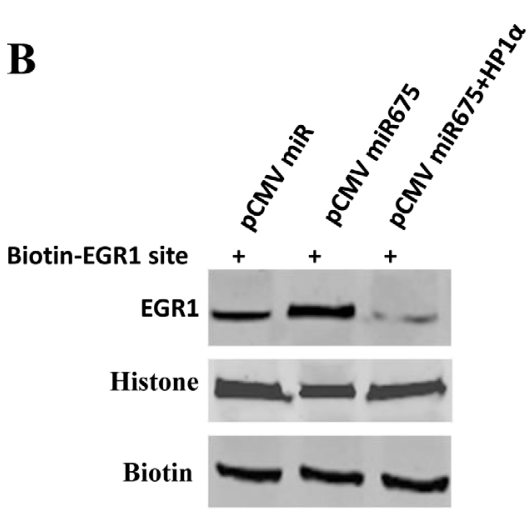

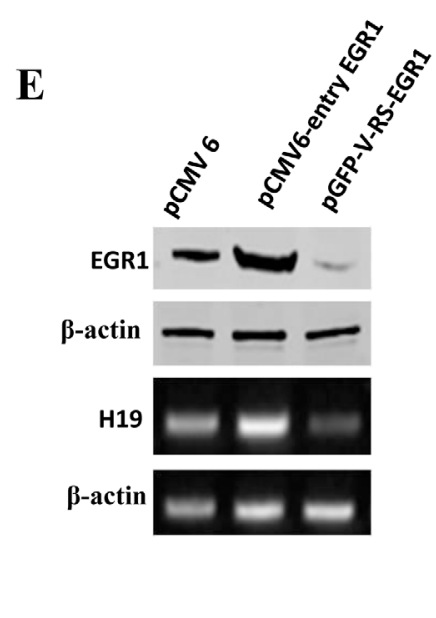

D

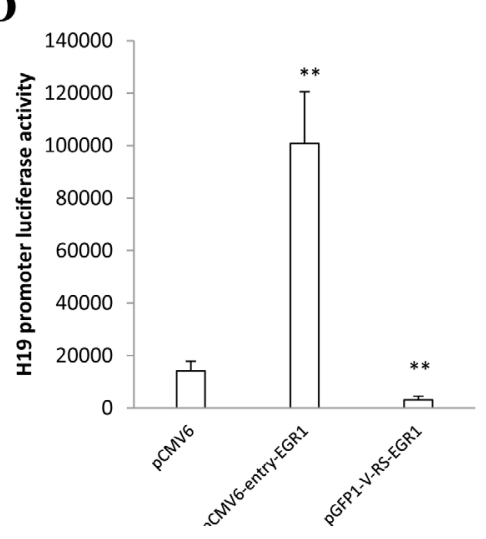




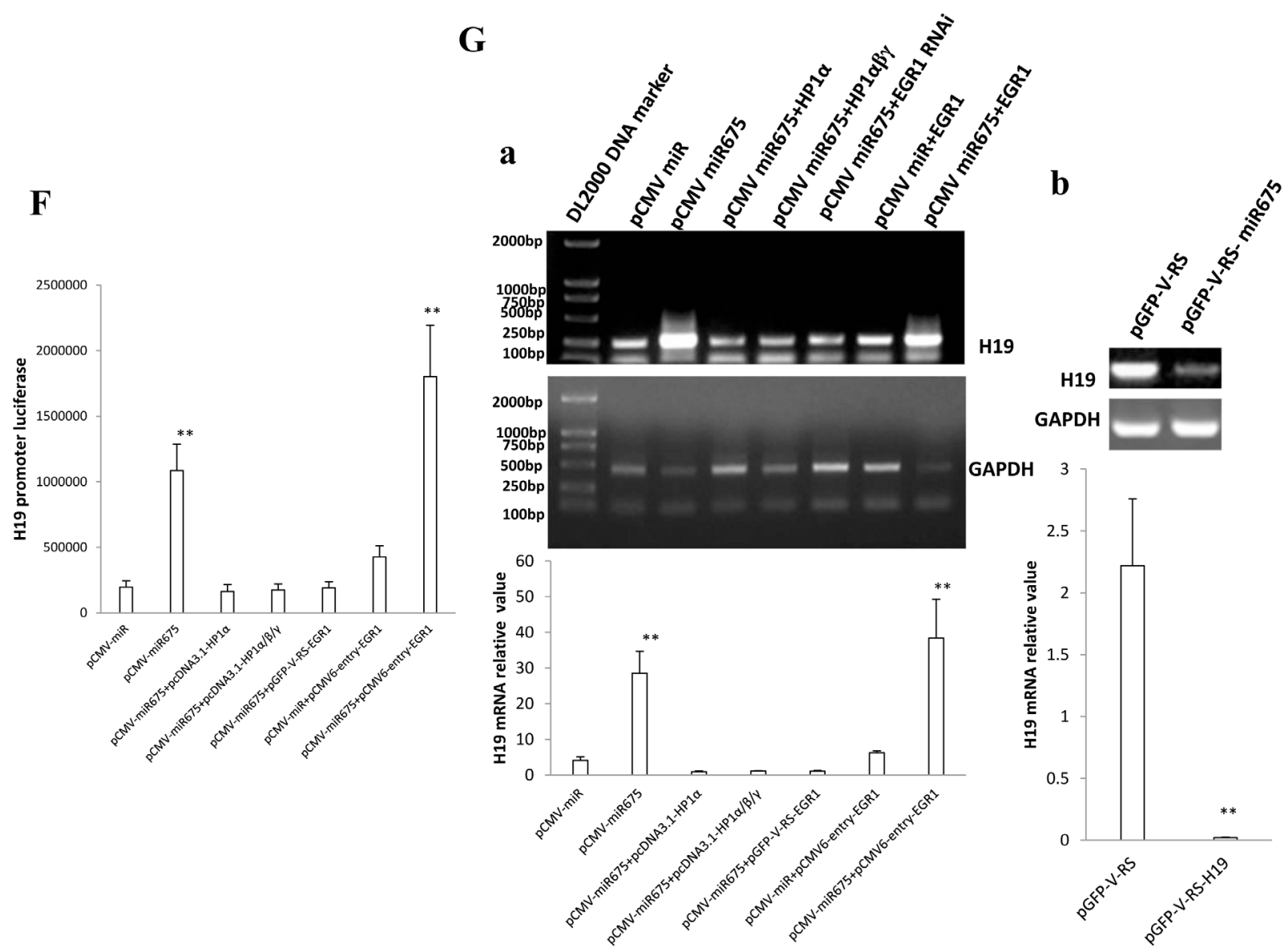

Figure 5: miR675 upregulates H19 through EGR1 activation. A. informatices analysis:EGR1 site (5'-CGCCCCCGC-3') and 5'-GCGGGGGCG-3' on H19 promoter region. B. DNA pulldown with Biotin-EGR1 followed by Western blotting with anti-EGR1in Hep3B transfected with pCMV-miR, pCMV-miR675, pCMV-miR675 plus pcDNA3.1-HP1 $\alpha$.Histone as internal control.Biotin as INPUT. C. Chromatin Immunoprecipitation (CHIP) with anti-EGR1 followed by PCR with H19 promoter primers in Hep3B transfected with pCMVmiR, pCMV-miR675, pCMV-miR675 plus pcDNA3.1-HP1 $\alpha$. IgG IP as negative control. H19 promoter DNA sequence as INPUT. D. H19 promoter luciferase activity assay in Hep3B cells transfected with pCMV6-entery, pCMV6-entry-EGR1, pGFP-V-RS-EGR1 respectively. Each value was presented as mean \pm standard error of the mean (SEM). E. Western blotting for EGR1 and RT-PCR analysis for H19 in Hep3B transfected with pCMV6-AC-GFP, pCMV6-AC-GFP-EGR1, pGFP-V-RS-EGR1 respectively. $\beta$-actin as internal control. F. H19 promoter luciferase activity assay in Hep3B transfected with pCMV-miR, pCMV-miR675, pCMV-miR675 plus pcDNA3.1HP1 $\alpha$, pCMVmiR675 plus pcDNA3.1HP1 $\alpha \beta \gamma$, pCMV-miR675 plus pGFP-V-RS- EGR1, pCMV-miR plus pCMV6-entry-EGR1, pCMV-miR675 plus pCMV6-entry-EGR1 respectively. Each value was presented as mean \pm standard error of the mean (SEM). G. a.RT-PCR analysis for H19 in Hep3B transfected with pCMV-miR, pCMV-miR675, pCMV-miR675 plus pcDNA3.1HP1 $\alpha$, pCMV-miR675 plus pcDNA3.1HP1 $\alpha \beta \gamma$, pCMV-miR675 plus pGFP-V-RS- EGR1, pCMV-miR plus pCMV6-entry-EGR1, pCMV-miR675 plus pCMV6-entry-EGR1 respectively. $\beta$-actin as internal control. b. RT-PCR analysis for H19 in Hep3B transfected with pGFP-V-RS, pGFP-V-RS--miR675 respectively. $\beta$-actin as internal control.

HP1 $\alpha$ compared to control. In addition, as shown in Figure 5D, H19 promoter luciferase activity was significantly increased in Hep3B cell line transfected with pCMVentry-EGR1 compared to control (100779.1 \pm 19715.4 vs 14039.0 $\pm 3835.9, P<0.01)$. However, H19 promoter luciferase activity was significantly decreased in Hep3B cell line transfected with pGFP-V-RS-EGR1 compared to control $(3058.7 \pm 1442.5$ vs $14039.0 \pm 3835.9, P<0.01)$. Further on, as shown in Figure 5E, both EGR1 expression. and H19 expression were significantly increased in Hep3B cell line transfected with pCMV-entry-EGR1 compared to control. In contrary, both EGR1 expression. and H19 expression were significantly decreased in Hep3B cell line transfected with pGFP-V-RS-EGR1 compared to control. It suggests EGR1 can enhance the H19 transcriptional activity. Strikingly, H19 promoter luciferase activity was significantly increased in Hep3B cells transfected with pCMV-miR675 (1084247.1 $\pm 201941.8 \quad$ vs 195688.2 $\pm 47675.9, \quad P<0.01), \quad$ pCMV-miR675 plus pCMV6-entry-EGR1 (1802573.2 \pm 389872.4 vs 195688.2 $\pm 47675.9, P<0.01)$ and pCMV-miR plus EGR1 (426217.7 \pm 85.85 .2 vs 195688.2 $\pm 47675.9, P<$ $0.01)$ compared to control. However, H19 promoter luciferase activity was significantly not altered in Hep3B 
cells transfected with pCMV-miR675 plus pcDNA3.1-

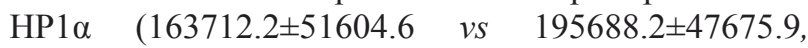
$P>0.05)$, pCMV-miR675 plus pcDNA3.1-HP1 $\alpha$, $\beta, \quad \gamma(174387.3 \pm 46190.9$ vs $195688.2 \pm 47675.9, \quad P>$ $0.05)$ and pCMV-miR675 plus pGFP-V-RS-EGR1 $(189216.3 \pm 47906.6$ vs $195688.2 \pm 47675.9, \quad P>0.05)$ compared to control (Figure 5F). Finally, we performed the RT-PCR in liver cancer line. As shown in Figure 5Ga, the H19 mRNA expression was significantly increased in Hep3B cells transfected with pCMV-miR675 and pCMVmiR675 plus pCMV6-entry-EGR1 compared to control. However, H19 mRNA was significantly not altered in Hep3B cells transfected with pCMV-miR675 plus pcDNA3.1-HP1 $\alpha$, pCMV-miR675 plus pcDNA3.1-HP1 $\alpha$, $\beta$, $\gamma$ and pCMV-miR675 plus pGFP-V-RS-EGR1 compared to control. Moreover, the H19 mRNA expression was significantly drcreased in Hep3B cells transfected with pGFP-V-RS-miR675 compared to pGFP-V-RS control (Figure 5Gb).Collectively, these observations strongly suggest that miR675 promotes H19 expression through upregulating and activating EGR1 by targeting for HP1 isoforms.

\section{miR675 accelerates hepatocarcinogenesis via miR675-HP1 $\alpha$-EGR1-H19-PKM2 axis}

Given that pre-miR675 is embedded in H19's first exon, we should consider whether ectopic overexpressed H19 can produce mature miR675. However, as shown in Figure 6Aa, mature miR675 turnout was significantly decreased in Hep3B cells transfected with pCI-H19 compared to control $(P<0.01)$. As shown in Figure 6Ab, mature miR675 turnout was significantly not altered in HepG2 cells transfected with pCI-H19 or pGFP-VRS-H19 compared to control $(P<0.01)$. It means that H19 may not act as a miR675 precursor. To identify whether miR675 oncogenic activity was caused by H19, we further determine the H19 function in liver cancer cells. In Hep3B cell line transfected with pCI-H19, the H19 expression was significantly enhanced in the Hep3B transfected with pCI-H19 compared to control (Figure 6B).Next, we performed the cell growth assay in vitro and colony formation ability test in liver cancer cell line. As shown in Figure $6 \mathrm{C}$, cell proliferation capacity was significantly improved in Hep3B cell line transfected with pCI-H19 compare to control in the second, third and fourth day, respectively $(P<0.01)$. As shown in the Figure 6D, Cell colony formation rate

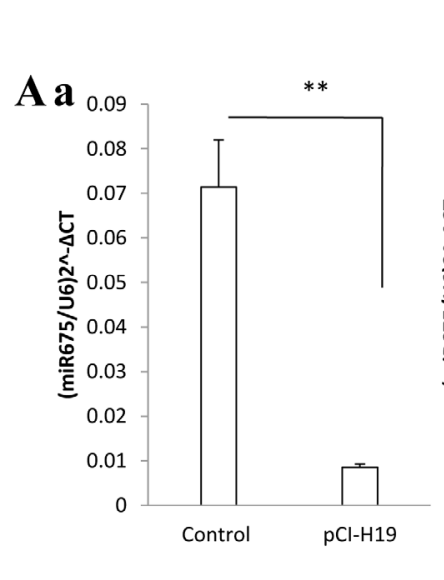

D

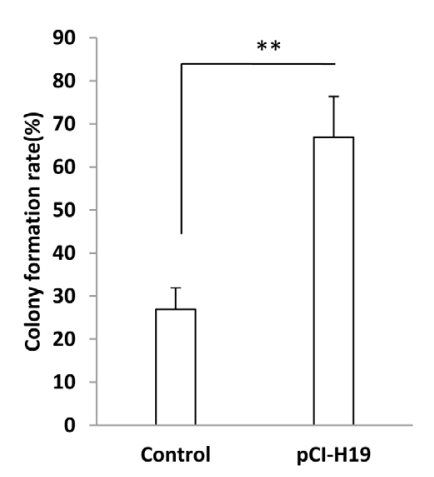

B

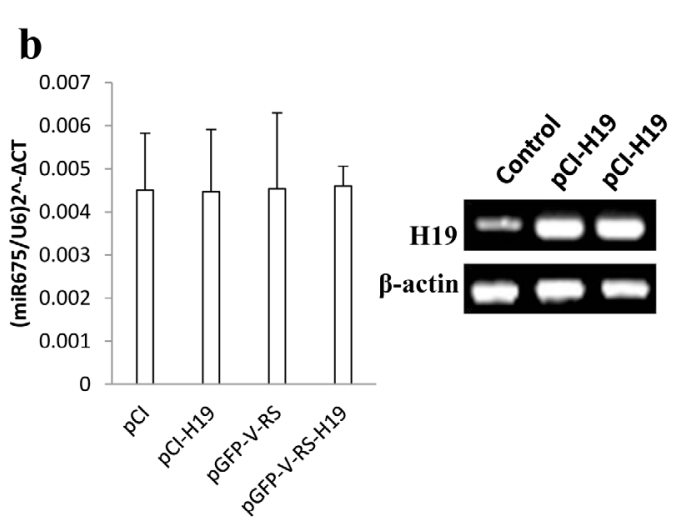

E

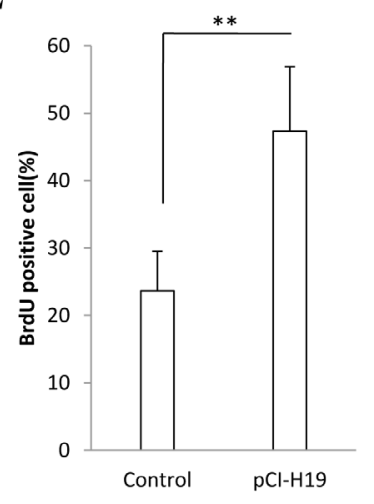

C

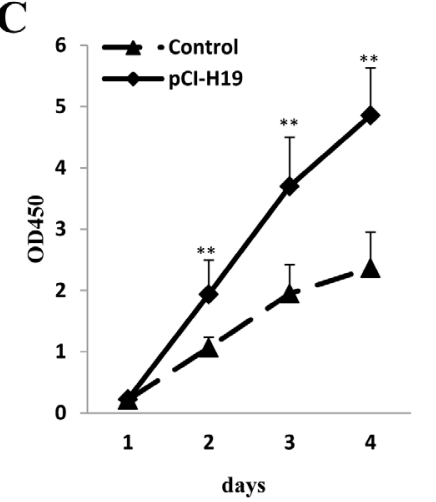

$\mathbf{F}$

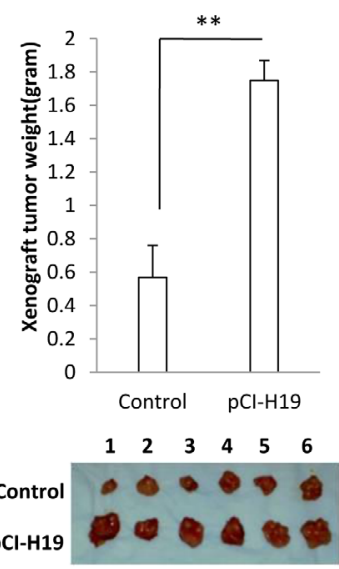



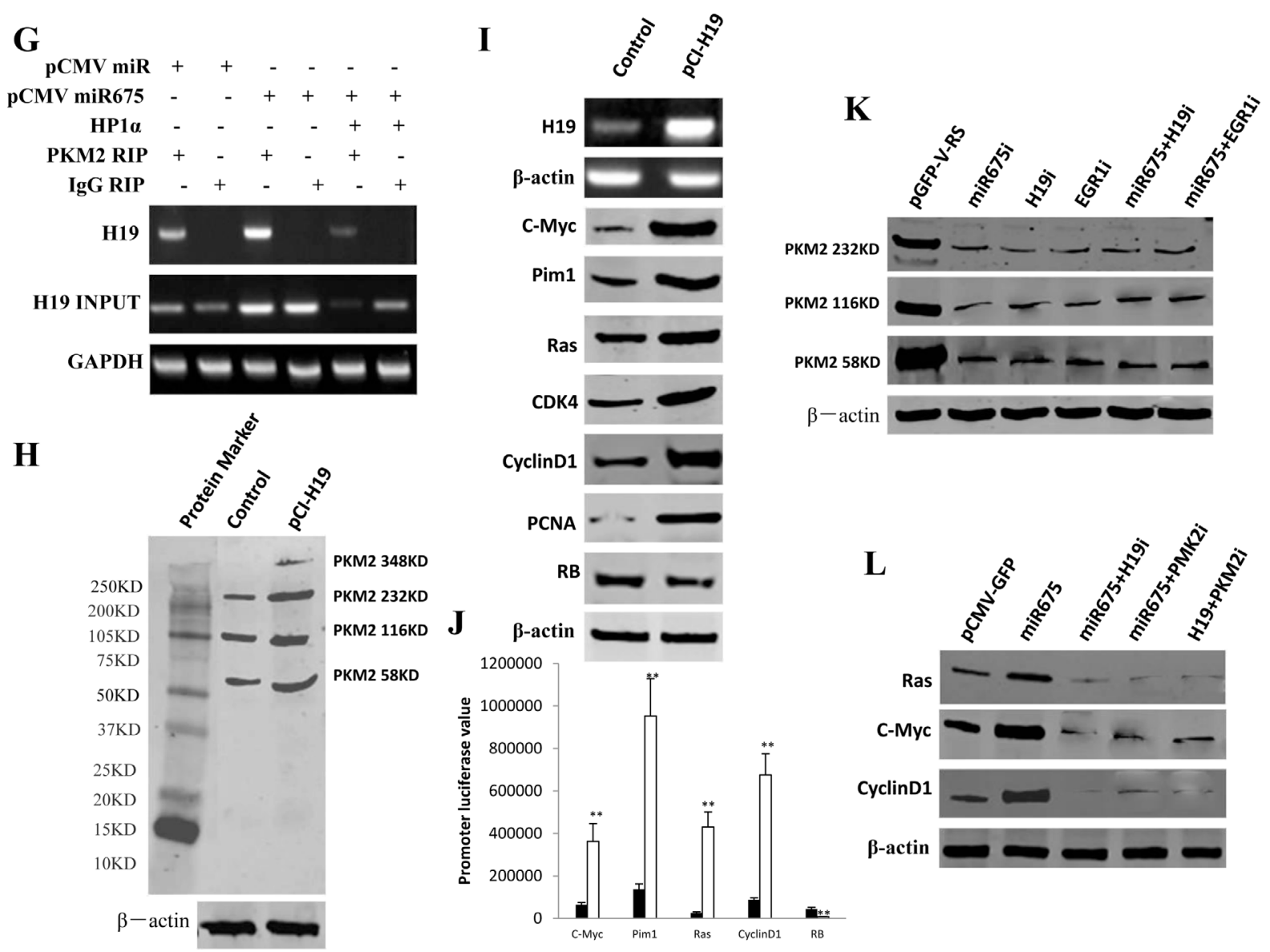

Figure 6: H19 upregulates and activates PKM2 to promote hepatocarcinogenesis A. a.real-time RT-PCR analysis for mature miR675 in Hep3B transfected with pCI-control, pCI-H19. U6 as internal control. b. real-time RT-PCR analysis for mature miR675 in HepG2 transfected with pCI-control, pCI-H19, pGFP-V-RS, pGFP-V-RS-H19 . U6 as internal control. B. RT-PCR analysis for H19 in Hep3B transfected with pCI-H19. $\beta$-actin as internal control. C. Cell proliferation assay was performed in 96-well format using the CCK8 cells proliferation kit to determine the cell viability as described by the manufacturer. Each sample was assayed in triplicates for 3 days consecutively. Cell growth curve was based on the corresponding the relative values of OD450 and each point represents the mean of three independent samples. Data are means of value from three independent experiments, bar \pm SEM. **, $P<0.01 ; *, P<0.05$. D. Cell plate colony formation ability assay. Data are means of value from three independent experiment, bar \pm SEM. $* *, P<0.01 ; *, P<0.05$. E. S phase cells assay using BrdU. Each value was presented as mean \pm standard error of the mean (SEM). F. Tumorigenesis test in vivo. Xenograft weight are means of value from six Balb/C nude mice, bar \pm SEM. ${ }^{*}, P<0.01 ; *, P<0.05$. G. RNA Immunoprecipitation (RIP) with anti-PKM2 followed by RT-PCR with H19 mRNA in Hep3B transfected with pCMV-miR, pCMV-miR675, pCMV-miR675 plus pcDNA3.1-HP1 $\alpha$. IgG IP as negative control. H19 cDNA sequence as INPUT. H. Western blotting analysis for PKM2 and its polymer in Hep3B transfected with pCI--H19. $\beta$-actin as internal control. I. Western blotting analysis for C-Myc, Pim1, H-Ras, CDK4, CyclinD1, RB and PCNA analysis in Hep3B transfected with pCI-H19. $\beta$-actin as internal control. J. C-myc, Ras, Pim1, CyclinD1 and pRB promoter luciferase activity assay in Hep3B transfected with pCI-H19 respectively. Each value was presented as mean \pm standard error of the mean (SEM). K. Western blotting analysis for PKM2 and its polymer in Hep3B transfected with pGFP-V-RS, pGFP-V-RS-miR675, pGFP-VRS-H19, pGFP-V-RS-EGR1, pCMV-miR675 plus pGFP-V-RS-H19, pCMV-miR675 plus pGFP-V-RS-EGR1. $\beta$-actin as internal control. L. Western blotting analysis for H-Ras, C-Myc, CyclinD1 in Hep3B transfected with pCMV-mir, pCMV-miR675, pCMV-miR675 plus pGFP-V-RS-H19, pCMV-miR675 plus pGFP-V-RS-PKM2, pCI-H19 plus pGFP-V-RS-PKM2. $\beta$-actin as internal control.

was significantly higher in Hep3B cell line transfected with pCI-H19 than in control $(66.9 \pm 9.4 \%$ vs $26.9 \pm 4.9$ $\%, P<0.01)$. BrdU positive cells rate was significantly higher in $\mathrm{H} 19$ overexpressed Hep3B cell line than that in control (47.4 $\pm 9.5 \%$ vs $23.7 \pm 5.8 \%, P<0.01$ ) (Figure $6 \mathrm{E})$. Importantly, xenograft tumor was greater in H19 overexpressed group than that in control $(1.75 \pm 0.12$ gram vs $0.57 \pm 0.19 \mathrm{gram}, P<0.01$ ) (Figure $6 \mathrm{~F}$ ). Intriguingly, as shown in Figure $6 \mathrm{G}$, the interaplay between PKM2 and H19 mRNA was significantly intensified in Hep3B cell line transfected with pCMV-miR675 compared to control. However, the interplay between PKM2 and H19 mRNA was significantly reduced in Hep3B cell line transfected with pCMV-miR675 plus pcDNA3.1-HP1 $\alpha$ compared to control. It may be caused by the reduced H19. Meaningfully, the PKM2 monomer (58KD), PKM2 dimer (116KD), PKM2 tetramer (232KD), PKM2 hexamer (348KD) were significantly increased in Hep3B cell line 
transfected with pCI-H19 compared to control respectively (Figure 6H).Ultimately, our results showed that C-Myc, Pim1, H-Ras, CDK4, CyclinD1, PCNA were increased and RB was decreased in H19 overexpressed Hep3B cell lines compared to control (Figure 6I). Moreover, C-myc (362168.7 \pm 84322.7 vs $64518.3 \pm 9667.5, P<0.01)$, pim 1 (952907.3 \pm 175384.8 vs $13625.0 \pm 26379.8, P<0.01)$, Ras (429923.7 \pm 71421.3 vs $25787.1 \pm 4911.64, P<0.01)$, CyclinD1 (675313.3 \pm 98493.1 vs $87377.7 \pm 8480.7, \quad P$ $<0.01$ ) promoter luciferase activity were significantly increased and RB promoter luciferase activity (7554.3 \pm 1329.4 vs $45125.2 \pm 6395.7, P<0.01$ ) were significantly decreased in H19 overexpressed Hep3B cell lines compared to control (Figure 6J). Further on, the PKM2 monomer (58KD), PKM2 dimer (116KD), PKM2 tetramer (232KD) were significantly decreased in Hep3B cell line transfected with pGFP-V-RS-miR675, pGFP-V-RS-H19, pGFP-V-RS-EGR1, pCMV-miR675 plus pGFP-V-RS-H19, pCMV-miR675 plus pGFP-V-RSEGR1 compared to control respectively (Figure 6K).On the other hand, our results showed that C-Myc, H-Ras, CyclinD1 were increased Hep3B cell lines transfected with pCMV--miR675 and decreased in Hep3B transfected with pCMV-miR675 plus pGFP-V-RS-H19, pCMVmiR675 plus pGFP-V-RS-PKM2, pCI-H19 plus pGFP-VRS-PKM2 compared to control respectively (Figure 6L).

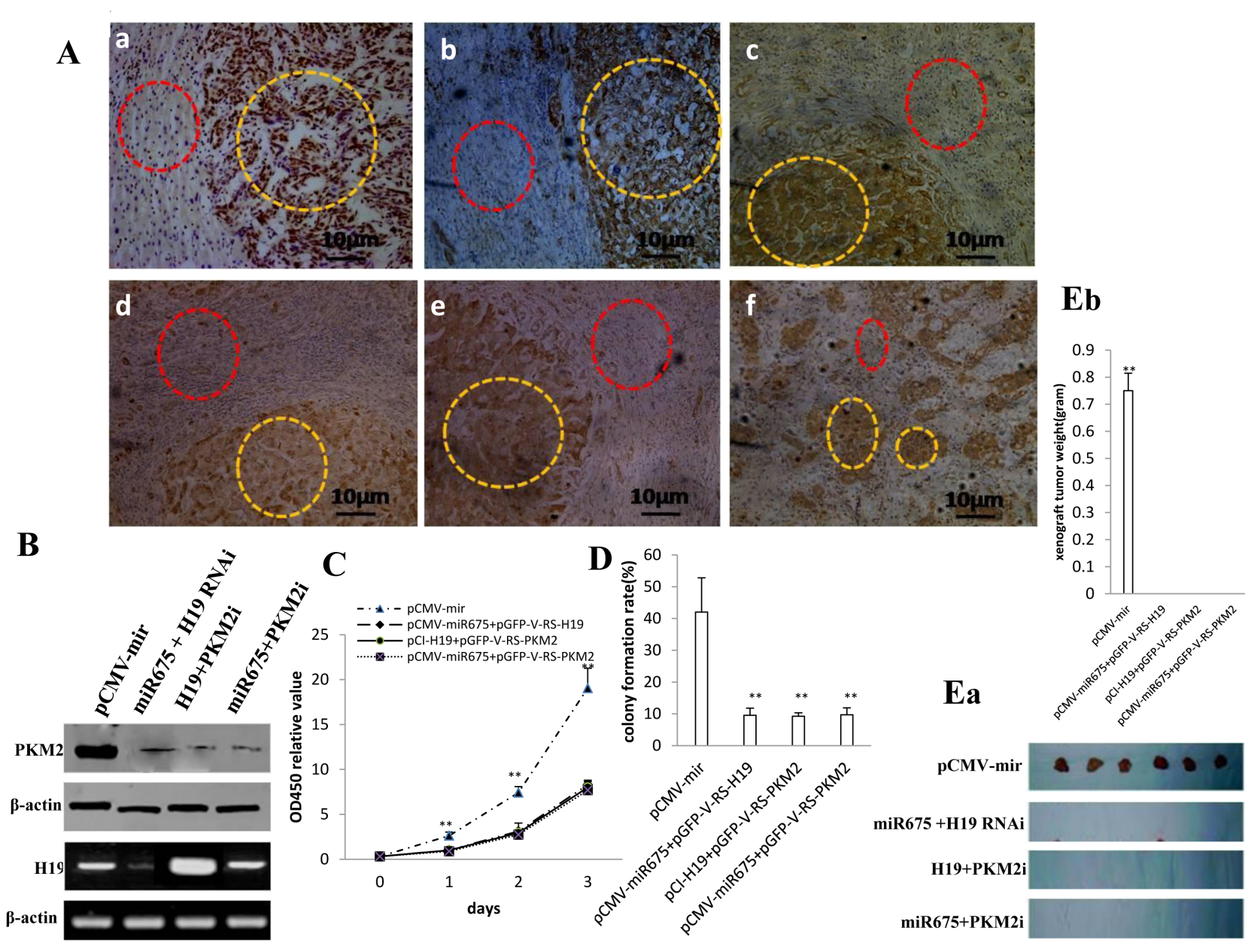

Figure 7: miR675 oncogenic action depends on PKM2 activity A. The representative analytic results of in situ hybridization for H19 and miR675, and immunohistochemistry staining for PKM2, HP1 $\alpha$, EGR1 in formalin-fixed, paraffin-embedded human liver cancer tissue (indicated with yellow Dotted circles) and their paired adjacent noncancerous tissues (indicated with Dotted red circles) from the same patient (DAB stainning, original magnification $\times 100$ ). B. Western blotting for PKM2 and RT-PCR analysis for H19 in HepG2 cell lines transfected with pCMV-miR, pCMV-miR675 plus pGFP-V-RS-H19, pCMV-miR675 plus pGFP-V-RS-PKM2, pCCI-H19 plus pGFP-VRS-PKM2. C. Cell proliferation assay was performed in 96-well format using the CCK8 cells proliferation kit to determine the cell viability as described by the manufacturer. Each sample was assayed in triplicates for 3 days consecutively .Cell growth curve was based on the corresponding the relative values of OD450 and each point represents the mean of three independent samples. Data are means of value from three independent experiments, bar \pm SEM. **, $P<0.01 ; *, P<0.05$. D. Cell plate colony formation ability assay.Data are means of value from three independent experiment, bar \pm SEM. $* *, P<0.01 ; *, P<0.05$. E. a. The photography of xenograft tumors from Balb/C nude mice injected with HepG2 cells transfected with pCMV-mir, pCMV-miR675 plus pGFP-V-RS-H19, pCMV-miR675 plus pGFP-VRS-PKM2, pCI-H19 plus pGFP-V-RS-PKM2 subcutaneously at armpit .b. The xenograft tumors weight (gram) in two groups indicated in left. Data were means of value from six Balb/C nude mice, mean $\pm \mathrm{SEM}, \mathrm{n}=6,{ }^{*}, P<0.05 ; * *, P<0.01$. 
Taken together, miR675 upregulates H19 that accelerates hepatocarcinogenesis through activating PKM2 to alter oncogenes expression and function positively.

\section{miR675 oncogenic function depends on activity of PKM2}

Given that miR675 activates PKM2 through H19 in liver cancer cell, we should consider whether miR675 oncogenic function depends on activity of PKM2. At the first time, our results showed that the expression level of miR675, H19, HP1 $\alpha$, EGR1, PKM2, H-Ras were consistent in 10 cases of liver cancer patients and their upregulated expression rate added up to $100 \%$ (liver cancer tissue $v s$ paracancerous liver tissue) (Figure 7A). Next, we analysed the PKM2 and H19 expression in stable HepG2 cell lines. PKM2 expression was decreased in stable HepG2 cell lines transfected with pCMV-miR675 plus pGFP-V-RS-H19, pCMV-miR675 plus pGFP-VRS-PKM2, pCI-H19 plus pGFP-V-RS-PKM2 compared to $\mathrm{pCMV}$-miR control. H19 expression was decreased in stable Hep3B cell lines transfected with pCMV-miR675 plus pGFP-V-RS-H19 and increased in stable HepG2 cell line pCI-H19 plus pGFP-V-RS-PKM2 (Figure 7B). Cell proliferation ability was significantly reduced in stable HepG2 cell lines transfected with pCMVmiR675 plus pGFP-V-RS-H19, pCMV-miR675 plus pGFP-V-RS-PKM2, pCI-H19 plus pGFP-V-RS-PKM2 compared to $\mathrm{pCMV-miR}$ control $(P<0.01)$ (Figure 7C).Cell colony formation ability was significantly decreased in stable HepG2 cell lines transfected with pCMV-miR675 plus pGFP-V-RS-H19, pCMV-miR675 plus pGFP-V-RS-PKM2, pCI-H19 plus pGFP-V-RSPKM2 compared to pCMV-miR control $(9.55 \pm 2.25 \%$, $9.26 \pm 1.06 \%, 9.72 \pm 2.17 \%$ vs $42.04 \pm 10.77 \%, P<0.01)$ (Figure 7D). Strikingly, xenograft tumors were produced only in stable HepG2 cell lines transfected pCMV-miR control (Figure 7Ea) and the xenograft average weight added up to $0.75 \pm 0.064$ gram $(n=6, P<0.01)$ (Figure $7 \mathrm{~Eb})$. Obviously, once PKM2 activity was lost, the tumorigenesis ability of miR675 was caused. Together, these observations suggest PKM2 determines the miR675 oncogenic action partly, at least in the human liver cancer cells.

\section{DISCUSSION}

It is well known that microRNAs (miRNAs) are short non-coding RNAs that are involved in post-transcriptional regulation of gene expression in multicellular organisms by affecting both the stability and translation of mRNAs. Our studies are now indicated to evaluate the effects of miR675 in liver cancer (Figure 8). Our present findings clearly demonstrate that miR675 overexpression promotes and silencing miR675 attenuated liver cancer cell growth in vitro and in vivo. To our knowledge, this is the first report demonstrating miR675 plays a positive role in liver carcinogenesis through the cascade of miR675-HP1 $\alpha$-EGR1-H19-PKM2 signaling. To this data, we report that the upregulated expression level of miR675, H19, HP1 $\alpha$, EGR1, PKM2, H-Ras were consistent in liver cancer patients and miR-675 upregulates long noncoding RNA H19 through activating EGR1 in human liver cancer cells. Strikingly, we confirm how miR675-HP1 $\alpha$-EGR1-H19-PKM2 cascade might be played an important role in hepatocarcinogenesis. On the other hand, we also proposed a key role for the miR675 in upregulation of $\mathrm{H} 19$ that may induce and activate PKM2, in turn, responsible for changes in gene expression relevant in hepatocarcinogenesis (e.g.C-myc, Pim1, Ras, CyclinD1, RB1).Obviously, this is a new linkage of miR675-HP1 $\alpha$-ERG1-H19-PKM2 in human liver cancer.

It is worth mentioning that miR675 play an important role in the occurrence of hepatocellular carcinoma. In this report, we focused mainly on the view that miR675 promotes human hepatocarcinogenesis by activating PKM2 dependent on the reduction of HP1 isoforms and the increase of H19. To date, accumulating evidence indicates that miR675 plays a critical role in cancer development and miR675 possess a strong carcinogen properties. Actually, our observations are consistent with these previous reports. For examples, miR-675 modulates human gastric cancer cell proliferation by targeting tumor suppressor Runt Domain Transcription Factor1 (RUNX1) [50];miR-675 was found to be upregulated in human colorectal cancer (CRC) tissues and the tumor suppressor retinoblastoma (RB) was confirmed to be a direct target of miR-675 [51]. Overexpression of the miR-675 in hepatocellular carcinoma links a dramatic upregulation of proliferative and growth capacity [52].Herein, our results showed that miR675 promotes hepatocarcinogenesis and progress through miR675-HP1 $\alpha$-EGR1-H19-PKM2 cascade signaling pathway. The involvement of miR675 promotion of liver cancer cell growth is supported by results from four parallel sets of experiments: (1)The upregulated expression level of miR675, H19, HP1 $\alpha$, EGR1, PKM2, H-Ras were consistent in liver cancer patients ; miR675 promotes the liver cancer cells malignant proliferation in vitro and accelerates liver cancer growth in vivo. (2) Mechanistically, miR675 inhibits the heterochromatin1 isoforms (HP1 $\alpha, \mathrm{HP} 1 \beta, \mathrm{HP} 1 \gamma)$ expression in human liver cancer cells which causes a dramatically decrease of the total histone $\mathrm{H} 3$ lysine 9 trimethylation (H3K9me3), histone $\mathrm{H} 3$ lysine 27 trimethylation (H3K27me3) and a increase of histone $\mathrm{H} 3$ lysine 27 acetylation (H3K27Ac); (3) Next, a significant reduction of the $\mathrm{H} 3 \mathrm{~K} 9 \mathrm{me} 3$ and H3K27me3 and the increment of H3K27Ac occupancy on the promoter region of EGR1 triggers EGR1 transcription, sumoylation and activation which upregulates lincRNA H19; (4)Ultimately, H19 may induce and activate tumor- 
specific pyruvate kinase M2 (PKM2) which is essential for the warburg effect in its dimer and for gene expression in its teramer during tumorigenesis. Evidently, miR675 is crucial for cell growth and viability in liver cancer cells. According to the aforementioned findings and reports, it is thus clear that miR675 has a strong carcinogenic ability.

It has been confirmed that heterochromatin protein
1 is a conserved eukaryotic chromosomal protein that is prominently associated with pericentric heterochromatin and mediates the concomitant gene silencing. Mechanistic studies implicate HP1 family proteins as 'hub proteins,' able to interact with a variety of chromosomal proteins through the chromo-shadow domain (CSD), as well as to recognize key histone modification sites [primarily

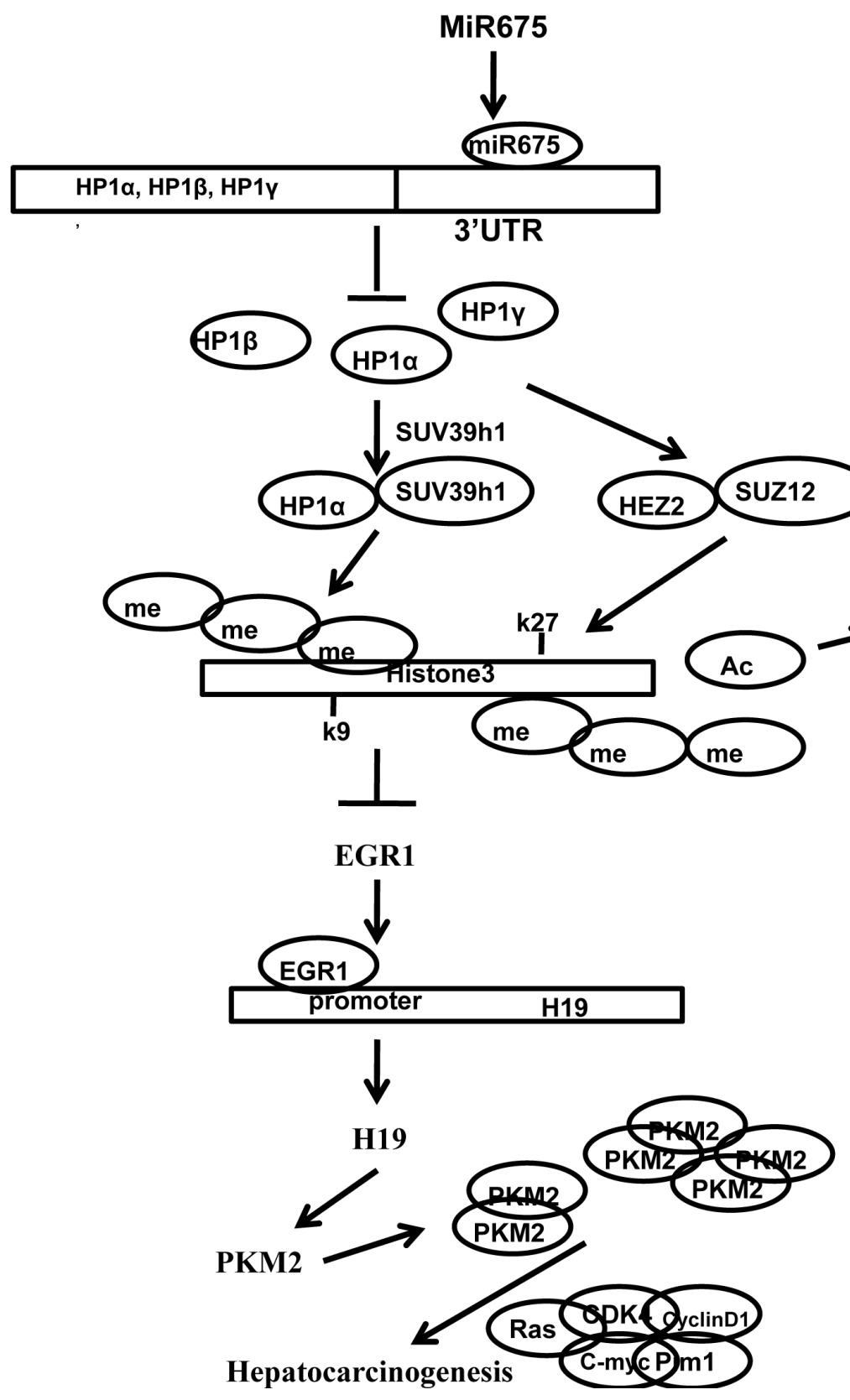

Figure 8: The schematic illustrates a model that miR675 is involved in the epigenetic regulation of H3K9me3 , H3K27me3 for gene expression in tumorigenesis. miR675 overexpression promotes and silencing miR675 attenuated liver cancer cell growth in vitro and in vivo. Mechanistically, miR675 decreases the heterochromatin protein HP1 $\alpha$, HP $1 \beta$, HP $1 \gamma$ expression in human liver cancer cells which causes a marginal decrease of the total histone H3 lysine 9 trimethylation (H3K9me3) and total histone H3 lysine 27 trimethylation (H3K27me3) and a increase of total histone $\mathrm{H} 3$ lysine 27 acetylation (H3K27Ac). Notably, a significant reduction of $\mathrm{H} 3 \mathrm{~K} 9 \mathrm{me} 3$ and H3K27me3 loading onto the promoter region of EGR1 which triggers EGR1 transcription, sumoylation and activation to upregulate H19, a large intergenic non-coding RNA (lincRNA).Intriguingly, H19 induces and activates tumor-specific pyruvate kinase M2 (PKM2) which is essential for the warburg effect in its dimer and for gene expression in its teramer during tumorigenesis. 
histone H3 di/trimethyl Lys9 (H3K9me2/3)] through the chromodomain (CD). HP1 function is altered by context, and potentially by post-translational modifications [53, 54]. For example, HP1 $\alpha$ interacts with the Suz12 subunit of the polycomb repressive complex 2 (PRC2) [55] and $\mathrm{H} 3$ tail cleavage could serve to release polycomb proteins from K27 methylated H3. A conformational switch in HP1 releases auto-inhibition to drive heterochromatin assembly heterochromatin protein 1 (HP1), which recognizes H3K9methylated chromatin, oligomerizes and forms a versatile platform that participates in diverse nuclear functions, ranging from gene silencing to chromosome segregation [56]. In this report, we identify heterochromatin protein 1 isoforms are valid targets of miR675 in liver cancer. Our data suggest that miR675 reduced the HP1 $\alpha$ expression on the transcriptional level through targeting for HP1 $\alpha$ 3 ' untranslational region. In particular, we also proved miR675 altered the epigenetic modifications on histone through HP1 $\alpha$ reduction. Further on, it lead to increase the early growth response gene1 (EGR1) turnout through transcriptional regulation based on reducing the complex output of HP1 $\alpha$-SUV39H1-Histone3 , HP1 $\alpha$-SUV39H1H3K27Ac, HP1 $\alpha$-SUZ12-EZH2 which caused the dramatically reduction of $\mathrm{H} 3 \mathrm{~K} 9 \mathrm{me} 3, \mathrm{H} 3 \mathrm{~K} 27 \mathrm{me} 3$ and the increment of H3K27Ac. Strikingly, it promoted the EGR1 occupancy on H19 imprinting control region (ICR) which ultimately enhances H19 expression, suggesting that HP1 isoforms HP1 $\alpha$ acts as the hub of miR675 epigenetic regulation function. In addition, our results showed that H19 promoter activity and H19 expression were highly enhanced in cells coexpressing with miR-675 and EGR1 expression when compared with cells expressing EGR1. It suggest EGR1 and miR-675 may function on H19 promoter in different pathways. On the other hand, It also suggest EGR1 can enhance miR675 function that triggers H19 transcription.

There is plenty of evidence that H19 is a oncogenic long noncoding RNA. For example, the levels of H19 was overexpressed in pancreatic ductal adenocarcinoma (PDAC) [57].H19 was shown to be regulated by c-Myc in Bcr-Abl-expressing cells. [58].H19 levels are remarkably increased in bladder cancer tissues, and upregulated H19 enhances bladder cancer metastasis by associating with EZH2 and inhibiting E-cad expression [59]. Epigenetic activation of the MiR-200 family contributes to H19-mediated metastasis suppression in hepatocellular carcinoma [60]. Polyploidization of murine mesenchymal cells is associated with suppression of the long noncoding RNA H19 and reduced tumorigenicity [61]. Up-regulated long non-coding RNA H19 contributes to proliferation of gastric cancer cells [62].Although increment of H19 may partly contribute to miR675 medicated promotion of liver cancer cell growth, our findings in this study provide novel evidence for an active role of H19 in miR675-mediated promotion of liver cancer cell growth. This assertion is based on several observations: (1)
miR675 enhanced the H19 transcription although ectopic H19 may not produce the mature miR675. (2) H19 can accelerate hepatocarcinogenesis through activating PKM2 to alter oncogenes expression and function, e.g.Cmyc, H-ras, pim1, CDK4, CyclinD1, RB. (3)miR675 oncogenic function will be abrogated if H19 is knocked down in liver cancer cells. These findings are noteworthy that $\mathrm{H} 19$ decides in miR675 oncogenic action through mediating various biological processes including cell proliferation, differentiation. It is very clear that $\mathrm{H} 19$ is connection of miR675-PKM2 axis that may contribute to hepatocarcinogenesis. It follows that H19 is a manipulator for miR675's oncogenic activity.

It is worth noting that PKM2 activity alteration is absorbing and of great concern. We observed that miR675 increased PKM2 expression and its activity through H19. Many cancer cells have increased rates of aerobic glycolysis, a phenomenon termed the Warburg effect. PKM2 expression was shown to be necessary for aerobic glycolysis and to provide a growth advantage to tumors. Knockdown of pyruvate kinase in tumor cells leads to a decrease in the levels of pyruvate kinase activity and an increase in the pyruvate kinase substrate phosphoenolpyruvate. PKM2 is dispensable for tumor maintenance and growth in vivo, suggesting that other metabolic pathways bypass its function [63]. PKM2 was frequently over-expressed in human HCC and its over-expression was associated with aggressive clinicopathological features and poor prognosis of HCC patients. Furthermore, knockdown of PKM2 suppressed aerobic glycolysis and cell proliferation in HCC cell lines in vitro. Importantly, knockdown of PKM2 hampered HCC growth in both subcutaneous injection and orthotopic liver implantation models, and reduced lung metastasis in vivo [64].Strikingly, PKM2 directly binds to histone $\mathrm{H} 3$ and phosphorylates histone $\mathrm{H} 3$ at $\mathrm{T} 11$ upon EGF receptor activation. PKM2 as a protein kinase in its nonmetabolic functions of histone modification is essential for its epigenetic regulation of gene expression and tumorigenesis [65]. PKM2 is upregulated in multiple cancer types and contributes to the Warburg effect by unclear mechanisms. Nuclear PKM2 acts as a coactivator of $\beta$-catenin to induce c-Myc expression, resulting in the upregulation of GLUT1, LDHA and, in a positive feedback loop [66]. It is evident that activation of PKM2 may play an important role in miR675 oncogenic action in liver cancer .Our findings in this study provide novel evidence for an active role of PKM2 in miR675-mediated promotion of liver cancer cell growth. This assertion is based on several observations: (1) miR675 upregulates H19, in turn, H19 increased the M2 isoform of pyruvate kinase (PKM2) expression and the formation of the PKM2 monomer, PKM2 dimer, PKM2 tetramer, PKM2 hexamer. (2)H19 and miR675 may activate PKM2. (3)Once PKM2 activity was lost, the tumorigenesis ability of H19 and miR675 was caused. PKM2 determines the miR675 and 
H19 oncogenic action partly, at least in liver cancer. It is suggest that miR675 and H19 tumorigenic action may require PKM2 participation. That is to say that PKM2 determines the carcinogenic effect of miR675 and H19. In view of this reason, we infer that miR675 and H19 may lead to PKM2 phosphorylation alternation, making PKM2 produce multimers.

In conclusion, we first proved that miR675 exerts its effect in part through the upregulation of H19 and PKM2 expression. Our present approaches provided an unequirocal evidence for critical oncogenic roles of the miR675 in hepaocarcinoma and supported the notion that miR675 may be an alternative bona fide promoting factor of hepatocarcinoma.We presented three miR675 novel mechanisms. Firstly, miR675 inhibits the HP1 isoforms expression and activates EGR1; Secondary, miR675 triggers EGR1 occupancy on $H 19$ promoter region and enhances H19 transcription positively; Thirds, miR675 controls PKM2 polymer formation dependent on H19 upregulation . On the basis of these mechanisms, miR675 exerts a tumorigenic functions through miR675-HP1 $\alpha$ EGR1-H19-PKM2 cascade signaling pathway in liver cancer. Although miR675 's oncogenic function was due to decrease the HP1 isoforms and increase H19, PKM2 in liver cancer cells, we further confirm how miR675-HP1EGR1-H19-PKM2 axis might be played an important role in hepatocarcinogenesis and progression. However, we have fully not understood the accuracy mechanism of miR675, such as, how miR675 controls HP1 isoforms dynamics change ? How miR675 regulates PKM2 polymer formation and drives the PKM2 from cytoplasmic yo nuclear? What are the recruitment factors, partners of miR675 during genes regulation and control? What is the clinic significance of miR675? etc. In this report, we focused mainly on the view that miR675 promotes human hepatocarcinogenesis by activating $\mathrm{H} 19$ dependent on reduction of HP1 isoforms. Our present findings open the possibility that targeting miR675 might prove to be an alternative therapeutic strategy, e.g.Baculovirus-mediated miRNA regulation to suppress hepatocellular carcinoma tumorigenicity and metastasis [67].It will produce an important implication for treatment and diagnosis of hepatocarcinoma. It is worth paying attention that we confirm that deciphering the molecular basis of miR675 in hepatocarcinogenesis is very important for us to apply miR675 in clinic diagnosis and therapy later.

\section{MATERIAL AND METHODS}

\section{Cell lines and plasmid}

Human hepatoma cell lines Hep3B and HepG2 were obtained from the Cell Bank of Chinese Academy of Sciences (Shanghai, China).These cell lines were maintained in Dulbecco's modified Eagle medium (Gibco BRL Life Technologies) supplemented with 10\% fetal bovine serum (sigma) in a humidified atmosphere of $5 \% \mathrm{CO}_{2}$ incubator at $37^{\circ} \mathrm{C}$. pCMV-miR, pCMV-miR675 (MI0005416), pGFP-V-RS, pCMV-AC-GFP, pCMV6entry-EGR1, pGFP-V-RS-EGR1 , pMitTarget were purchased from Origene (Rockville, MD, USA) and pcDNA3.1, pcDNA3.1-HA--HP1 $\alpha$, pcDNA3.1-HA-HP1 $\beta$, pcDNA3.1-HA--HP1 $\gamma$, pBS-H19, pGL3-C-myc, were purchased from Addgene (Cambridge MA, USA). pCI-H19, pMirTarget-HP1 $\alpha, \beta, \gamma 3$ 'UTR, pMirTargetmutant HP1 $\alpha, \beta$, $\gamma 3$ 'UTR, pCMV-mutant miR675, pGFPV-RS-mir675, pGFP-V-RS-H19 , pGFP-V-RS-PKM2 , pGL3-EGR1, pGL3-H-Ras, pGL3-CyclinD1, pGL3-Pim1, pGL3-RB was constructed by ourselves.

\section{Cell transfection and stable cell lines}

Hep3B or HepG2 cells were transfected using transfection reagent lipofectamine ${ }^{R} 2000$ (Invitrogen) according to manufacturer's instructions respectively. For screening miR675 overexpression or knockdown Hep3B or HepG2 stable cell lines, these cells were plated in the selective medium containing $2500 \mu \mathrm{g} / \mathrm{ml} \mathrm{G} 418$ (Invitrogen) or $1 \mu \mathrm{g} / \mathrm{ml}$ Puromycin (Calbiochem) in fortyeight hours after transfection. For the next 4-8 weeks, the selective media were replaced every 3 days. Once the distinct conoly of surviving cells were transferred into 96-well plate and continued to maintain cultures in selected media. Transfection efficiency was observed by GFP imaging and measured by real-time RT-PCR, Western blotting.

\section{Reverse-transcriptase polymerase chain reation}

Total RNA was purified using Trizol (Invitrogen) according to manufacturer's instructions . cDNA was prepared by using oligonucleotide $(\mathrm{dT})_{18}$ and a SuperScript First-Strand Synthesis System (Invitrogen).The PCR amplification kit (TaKaRa) were adopted according to the manufacturer's instructions. H19 cDNA was amplified using the upstream primer (5'-attgcgcagcaaggaggctg-3') and the down stream primer (5'-cctccctcctgagagctcat-3') (synthesized by Shenggong, Shanghai, China) under the PCR reaction conditions performed in 35 cycles with each cycle consisting of a denaturation step $\left(94^{\circ} \mathrm{C}\right.$ for 30 seconds, and 3 minutes for the first cycle only), an annealing step $\left(58^{\circ} \mathrm{C}\right.$ for 30 seconds) and an elongation step $\left(72^{\circ} \mathrm{C}\right.$ for 30 seconds, 10 minutes for the last cycle only ). $\beta$-actin served as a internal control for the efficiency of the RT-PCR. $\beta$-actin primer: P1:5' -gggaaatcgtgcgtgacatt3';P2:5' -ctcaggaggagcaatgatct-3'; Real-time PCR $\beta$ actin primer: $\mathrm{P} 1: 5^{\prime}$ '-ggtcatcaccattggcaatg-3';P2:5'aaggtagtttcgtggatgcc-3'.PCR products were analyzed by $1.0 \%$ agarose gel electrophoresis and visualized by 
ethidium bromide staining using Image imaging system (Baygene).

\section{MicroRNA detection}

Total RNA was isolated from cultured cells using Trizol (Invitrogen, Carlsbad, CA, USA) according to the manufacturer's protocol. Real-time RT-PCRbased detection of mature miR-675 and U6 snRNA was achieved with the miRNA Detection kit (including a universe primer, U6 primers, Qiagen) and miR675 specific upsteam primers (Origene, USA). qRT-PCR was performed with a StepOnePlus real-time PCR system (Applied Biosystems), a SuperScript First-Strand Synthesis System (Invitrogen, Carlsbad, CA, USA) and Power SYBR Green PCR Master Mix (Applied Biosystems) in accordance with the manufacturers' protocols. Each sample was run in triplicate. $C_{\mathrm{t}}$ values for miR675 were calculated and normalized to $C_{\mathrm{t}}$ values for U6 snRNA. The following primers were used: human mi675P1: 5'-TGGTGCGGAGAGGGC-3' and P2:5'- GAACATGTCTGCGTATCTC-3'. U6 primer:P1:5'-GCTTCGGCAGCACATATACT-3';P2:5'GGAACGCTTCACGAATTTGC-3'

\section{Western blotting}

The logarithmically growing cells were washed twice with ice-cold phosphate-buffered saline (PBS, Hyclone Lab. INC) and lysed in RIRP lysis buffer [50 mM Tris- $\mathrm{HCl}$ (pH 7.4), $150 \mathrm{mM} \mathrm{NaCl,} \mathrm{1 \%} \mathrm{NP-}$ $40, \quad 0.1 \%$ SDS] containing protease inhibitor cocktails (Roch, Diagnostics, Indianapolis IN, USA). Cells lysates were centrifuged at $12,000 \mathrm{~g}$ for 20 minutes at $4^{\circ} \mathrm{C}$ after sonication on ice, and supernatants were separated. After being boiled for 10 minutes in the presence of 2-mercaptoethanol, samples containing cells or tissue lysate proteins were separated on a $10 \%$ sodium dodecyl sulfate-polyacrylamide gel electrophoresis (SDSPAGE) and transferred onto a nitrocellulose membranes Membranes were stained and then blocked in 10\% dry milk-TBST [20mM Tris-HCl (PH 7.5)], 0.1\% Tween 20 ) for $1 \mathrm{~h}$ at $37^{\circ} \mathrm{C}$. Following three washes in Tris- $\mathrm{HCl}$ pH 7.5 with $0.1 \%$ Tween 20 , the blots were incubated with antibody (appropriate dilution) overnight at $4^{\circ} \mathrm{C}$. Following three washes, membranes were then incubated with secondary antibody for $60 \mathrm{~min}$ at $37^{\circ} \mathrm{C}$ or $4^{\circ} \mathrm{C}$ overnight in TBST. Signals were visualized by ODYSSEY infrared imaging system (LI-COR). Standard western immunoblotting procedures were used with the following antibodies: Rabbit polyclonal anti-HP1 $\alpha$, Rabbit polyclonal anti-HP1 $\beta$, Rabbit polyclonal anti-HP1 $\gamma$, Rabbit polyclonal anti-H3K9me3, Rabbit polyclonal anti-H3K27me3, Rabbit polyclonal anti-SUV39h1, Rabbit polyclonal anti-Histone3, Rabbit polyclonal anti-
pHistone3, Rabbit polyclonal anti-H3K4me3, Rabbit polyclonal anti-H3K27Ac, Rabbit polyclonal antiPKM2, Rabbit polyclonal anti-Pim1, mouse monoclonal anti-EGR1, mouse monoclonal anti-SUZ12, mouse monoclonal anti-EZH2, mouse monoclonal anti-CMyc, mouse monoclonal anti-Ras, mouse monoclonal anti-CDK4, mouse monoclonal anti-CyclinD1, mouse monoclonal anti-RB, mouse monoclonal anti-PCNA, mouse monoclonal anti- $\beta$-actin were purchased from Santa Cruz, Biotech. IRDye 680LT /IRDye 800CW secondary antibodies were purchased from LI-COR scientific company. All other reagents and compounds were analytical grades (Sigma, Promega, Shengong, etc).

\section{Co-immunoprecipitation (IP)}

Cells were lysed in RIRP lysis buffer containing protease inhibitor cocktails (Roch, Diagnostics, Indianapolis IN, USA). Five-hundred-microliter cell lysates was used in immunoprecipitation with antibody. In brief, protein was pre-cleared with $30 \mu \mathrm{l}$ protein G/Aplus agarose beads (Santa Cruz, Biotechnology, Inc.CA) for 1 hour at $4^{\circ} \mathrm{C}$ and the supernatant was obtained after centrifugation $(5,000 \mathrm{rpm})$ at $4^{\circ} \mathrm{C}$. Precleared homogenates (supernatant) were incubated with $2 \mu \mathrm{g}$ of antibody and/or normal mouse/rabbit IgG by rotation for 4 hours at $4{ }^{\circ} \mathrm{C}$, and then the immunoprecipitates were incubated with $30 \mu \mathrm{l}$ protein $\mathrm{G} / \mathrm{A}$-plus agarose beads by rotation overnight at $4^{\circ} \mathrm{C}$, and then centrifuged at $5000 \mathrm{rpm}$ for $5 \mathrm{~min}$ at $4^{\circ} \mathrm{C}$. The precipitates were washed five times $\times 10 \mathrm{~min}$ with beads wash solution ( $50 \mathrm{mM}$ pH7.6 TrisCl, $150 \mathrm{mMNaCl}$, $0.1 \% \mathrm{NP}-40,1 \mathrm{mM}$ EDTA) and then resuspended in $40 \mu \mathrm{l}$ $2 \times$ SDS-PAGE sample loading buffer to incubate for 10 $\min$ at $100^{\circ} \mathrm{C}$. Then Western blot was performed with a another related antibodies.

\section{DNA pull down}

Cells were lysed by sonication in HKMG buffer (10 mM HEPES, PH7.9, $100 \mathrm{mM} \mathrm{KCl,} 5 \mathrm{mM} \mathrm{MgCl}$, $100 \%$ glycerol, $1 \mathrm{mM}$ DTT, and $0.5 \%$ NP40) containing protease inhibitors for the preparation of nuclear exact. Equal amount of cell nuclear extracts were precleared with Streptavidin-agarose Resin (Thermo) for 1 hours, and then were incubated with $1 \mu \mathrm{g}$ biotinylated EGR1 site double-stranded-oligonucleotides (forward:5'biotin-cgececegecgececegc-3'and reverse: 5'-biotingcgggggcggcgggggce $-3^{\prime}$ ) and together with $10 \mu \mathrm{g}$ poly (dI-dC) at $4^{\circ} \mathrm{C}$ for 24 hours. DNA-bound proteins were collected with the incubation with streptavidinagarose Resin for 1 hour with gently shaking to prevent precipitation in solution. Following five times washings of the resin bound complex with $0.5-1.0 \mathrm{ml}$ of binding buffer, the samples were boiled and subjected to SDS-PAGE and Western blotting analysis. 


\section{Dual luciferase reporter assay}

Cells $\left(1 \times 10^{5} /\right.$ well of a six-well plate) were transiently transfected with $1.5 \mu \mathrm{g}$ of luciferase construct (pMirtargetHP1 $\alpha \beta \gamma 3$ 'UTR, pGL3-EGR1, pBS-H19, et al) or indicated plasmids and $0.2 \mu \mathrm{g}$ of pRL-tk (promega) with the use of the Lipofectiamine ${ }^{\mathrm{TM}} 2000$ (Invitrogen). Transfection was performed with at least three different batches of each reporter plasmid. After incubation for 36-48 hours, the cells were harvested with Passive Lysis Buffer (Promega), and luciferase activities of cell extracts were measured with the use of the Dual luciferase assay system (Promega) according to manufacturer's instructions. luciferase activity was measured and normalized for transfection efficiency with Renilla luciferase activity. Transfection was performed with at least three different batches of each reporter plasmid.

\section{Chromatin immunoprecipitation (CHIP)}

Cells were cross-linked with $1 \%$ (v/v) formaldehyde for $10 \mathrm{~min}$ at room temperature and stopped with $125 \mathrm{~mm}$ glycine for $5 \mathrm{~min}$. Crossed-linked cells were washed with phosphate-buffered saline, resuspended in lysis buffer, and sonicated for 8-10 $\mathrm{min}$ in a SONICS to generate DNA fragments with an average size of 500-1000 bp. Chromatin extracts were diluted 5-fold with dilution buffer, pre-cleared with Protein-A/GSepharose beads, and immunoprecipitated with specific antibody on Protein-A/G-Sepharose beads. After washing, elution and de-cross-linking, the ChIP DNA was detected by traditional PCR (36 cycles) on the PCR Detection system (Bio-Rad).The following primer pairs were used: CHIP EGR1 primer EGR1 promoter DNA (AJ243425) :P1: P1:5'-cagcaccttatttggagtgg-3' (20112030); P2:5'-acctccatcctgcagggtag-3' (2191-2210). CHIP EGR1 negative primer :P1:5'-aagctactcgagaggaggag-3' (183-202); P2:5' -agatggecttgtgtctgaat-3' (361-380) and H19/promoter: (P1:5'-tgtatttctggaggcttccc-3';P2: 5'-tcagacacgtagcccgatat-3').

\section{RNA immunoprecipitation (RIP)}

Cells were lysed $\left(15 \mathrm{~min}, 0^{\circ} \mathrm{C}\right)$ in $100 \mathrm{mM} \mathrm{KCl}$, $5 \mathrm{mM} \mathrm{MgCl}, 10 \mathrm{mM}$ HEPES [pH 7.0], 0.5\% NP40, $1 \mathrm{mM}$ DTT, 100 units/ml RNase OUT (Invitrogen), $400 \mu \mathrm{M}$ vanadyl-ribonucleoside complex and protease inhibitors (Roche), clarified and stored on at $-80^{\circ} \mathrm{C}$. Ribonucleoprotein particle-enriched lysates were incubated with protein A/G-plus agarose beads (Santa Cruz, Biotechnology, Inc.CA) together with antiPKM2 or normal mouse IgG for 4 hours at $4^{\circ} \mathrm{C}$. Beads were subsequently washed four times with $50 \mathrm{mM}$ Tris- $\mathrm{HCl}$ ( $\mathrm{pH} 7.0$ ), $150 \mathrm{mM} \mathrm{NaCl}, 1 \mathrm{mM} \mathrm{MgCl}_{2}$, and
$0.05 \%$ NP-40, and twice after addition of $1 \mathrm{M}$ Urea. Immunoprecipitates (IPs) were digested with proteinase $\mathrm{K}\left(55^{\circ} \mathrm{C} ; 30^{\prime}\right)$ and mRNAs were then isolated and purified. RT-PCR was performed with the primers as follows: H19/P1:5' -attgcgcagcaaggaggctg-3', H19/P2:5' cctccetcctgagagctcat- 3 '.

\section{Cells proliferation assay}

Cells at a concentration $4 \times 10^{3}$ were seeded into 96well culture plates in $100 \mu \mathrm{l}$ culture medium containing $10 \%$ fetal calf serum (FCS).Before detected, add $10 \mu \mathrm{g} /$ well cell proliferation reagent CCK8 (Yeasen) and incubate for 4 hours at $37^{\circ} \mathrm{C}$ and $5 \% \mathrm{CO}_{2}$. Measure the absorbance of the samples against a background control as blank using SpectraMax M5 (Molecular Devices, MD, USA) according to the manufacturer instruction. Each sample was assayed in triplicates at daily intervals after seeding for up to 3 days consecutively .Cell growth curve was based on the corresponding the normalized values of OD450 and each point represents the mean of three independent samples.

\section{Colony-formation efficiency assay}

$1 \times 10^{3}$ cells were plated on a $10 \mathrm{~cm}$ dish, the 10 $\mathrm{ml}$ DMEM containing 10\%FBS was added into each $10 \mathrm{~cm}$ dish of the three replicate. Then these dishes were incubated at $37^{\circ} \mathrm{C}$ in humidified incubator for 10 days. Cell colonies on the dishes were stained with $1 \mathrm{ml}$ of 0 . 5\% Crystal Violet for more than 1 hour and the colonies were counted.

\section{BrdU staining}

$80 \%$ confluent cells were cultured for 24 hour before treatment with $10 \mu \mathrm{l} \mathrm{BrdU}$ (Roche) for 4 hours. Immunofluorescent staining with an anti-BrdU antibody was performed according to the manufacturer's instructions (Becton Dickinson). BrdU positive cells from ten random chosen fields of at least three independent samples were counted.

\section{Immunohistochemistry}

Tissues were fixed with $4 \%$ paraformaldehyde, dehydrated, embedded in paraffin and sectioned at $4 \mu \mathrm{m}$. Sections were immunohistochemically stained using mouse anti-human monoclonal anti-PKM2, anti-H-Ras, anti-EGR1, anti-HP1 $\alpha$, (Santa Cruz, Biotech). As the secondary antibody, anti-mouse IgG (Horseradish peroxidase linked whole antibody from sheep, GE Healthcare Limited) was used at $100 \times$ dilution. Staining was performed using 3, 3-diaminobenzidine 
(DAB) substrate kit for peroxidase according to the manufacturer's instructions (Vector Laboratories Inc) and counterstained with hematoxylin.

\section{In situ hybridization}

For tissus slides, deparaffinization and antigen retrieval (Digest with $20 \mu \mathrm{g} / \mathrm{ml}$ proteinase $\mathrm{K}$ in prewarmed $50 \mathrm{mM}$ Tris for 10 to $20 \mathrm{~min}$ at $37^{\circ} \mathrm{C}$.). Rinse slides 5 times in distilled water. Immerse slides in ice cold 20\% (v/v) acetic acid for $20 \mathrm{sec}$. Dehydrate the slides by washing for approximately $1 \mathrm{~min}$ each wash in $70 \%$ ethanol, 95\% ethanol and 100\% ethanol then air dry.Add $100 \mu \mathrm{l}$ of hybridization solution to each slide.Incubate the slides for $1 \mathrm{hr}$ in a humidified hybridization chamber at the $42^{\circ} \mathrm{C}$. Under heat at $95^{\circ} \mathrm{C}$ for $2 \mathrm{~min}$, to denature the DIG (Digoxigenin) labeled DNA probe .Drain off the hybridization solution. Add $50 \mu$ of diluted probe per section. Incubate in the humidified hybridization chamber at 42 overnight. While incubating, the sample on the slide can be covered with a cover slip to prevent evaporation. Stringency washes: Wash 1: 50\% formamide / 2 x SSC (3 $\mathrm{x}$ for $5 \mathrm{~min}, 37-45^{\circ} \mathrm{C}$ ). Wash 2: $0.1-2 \mathrm{x} \mathrm{SSC} 3 \mathrm{x}$ for $5 \mathrm{~min}$, $25^{\circ} \mathrm{C}$ to $75^{\circ} \mathrm{C}$. Wash twice in MABT (maleic acid buffer containing Tween 20) for $30 \mathrm{~min}$ at room temperature. Dry the slides.Transfer to a humidified chamber and add $200 \mu \mathrm{l}$ blocking buffer to each section (MABT $+2 \%$ BSA, milk or serum). Block for 1 to 2 hours, at room temperature. Drain off the blocking buffer. Add the anti-DIG antibody at the required dilution in blocking buffer. Wash slides 5 times with MABT, $10 \mathrm{~min}$ for each wash, at room temperature. SABC-DAB staining and washing slides in distilled water. Air dry the slides for around $30 \mathrm{~min}$. Wash in $100 \%$ ethanol, then air dry thoroughly. Mount using DePeX mounting solution.

\section{Xenograft transplantation in vivo}

Four-weeks male athymic Balb/C mice were purchased from Shi Laike company (Shanghi, China) and maintained in the Tongji animal facilities approved by the China Association for accreditation of laboratory animal care. The athymic Balb/C mice were injected at the armpit area subcutaneously with suspension of $7 \times 10^{6}$ transfected Hep3B cells or HepG2 cells in $100 \mu \mathrm{l}$ of phosphate buffered saline. The mice were observed four weeks, and then sacrificed to recover the tumors. The wet weight of each tumor was determined for each mouse. A portion of each tumor was fixed in $4 \%$ paraformaldehyde and embedded in paraffin for histological hematoxylineosin (HE) staining and anti-PCNA immunohistochemical staining. The use of mice for this work was reviewed and approved by the institutional animal care and use committee in accordance with China national institutes of health guidelines.

\section{Statistical analysis}

The significant differences between mean values obtained from at least three independent experiments. Each value was presented as mean \pm standard error of the mean (SEM) unless otherwise noted, with a minimum of three replicates. The results were evaluated by SPSS20.0 statistical soft (SPSS Inc Chicago, IL) and Student's t-test , Chi-square test were used for comparisons, with $P<0.05$ considered significant.

\section{ACKNOWLEDGMENTS \& FUNDING}

This study was supported by grants from National Natural Science Fundation of China (NCSF, No.81272291) and Science and Technology Commission of Shanghai Municipality (No.13JC1405500-13JC1405501)

\section{CONFLICTS OF INTEREST}

The authors disclose no conflicts.

\section{REFERENCES}

1. Li D, Kang J, Golas BJ, Yeung VW, Madoff DC.Minimally invasive local therapies for liver cancer. Cancer Biol Med. 2014;11:217-36.

2. Keniry A, Oxley D, Monnier P, Kyba M, Dandolo L, Smits G, Reik W.The H19 lincRNA is a developmental reservoir of miR-675 that suppresses growth and Igflr. Nat Cell Biol. 2012;14:659-65.

3. Dudek KA, Lafont JE, Martinez-Sanchez A, Murphy CL. Type II collagen expression is regulated by tissue-specific miR-675 in human articular chondrocytes.J Biol Chem. 2010;285:24381-7.

4. Kim NH, Choi SH, Lee TR, Lee CH, Lee AY.Cadherin 11, a miR-675 target, induces $\mathrm{N}$-cadherin expression and epithelial-mesenchymal transition in melasma. J Invest Dermatol. 2014;134:2967-76.

5. Shi Y, Wang Y, Luan W, Wang P, Tao T, Zhang J, Qian J, Liu N, You Y. Long non-coding RNA H19 promotes glioma cell invasion by deriving miR-675.PLoS One. 2014;9:e86295.

6. Goodell MA. Parental permissions: H19 and keeping the stem cell progeny under control.Cell Stem Cell. 2013;13:137-8.

7. Zhu M, Chen Q, Liu X, Sun Q, Zhao X, Deng R, Wang Y, Huang J, Xu M, Yan J, Yu J.lncRNA H19/miR-675 axis represses prostate cancer metastasis by targeting TGFBI. FEBS J. $2014 ; 281: 3766-75$.

8. Gao WL, Liu M, Yang Y, Yang H, Liao Q, Bai Y, Li YX, Li D, Peng C, Wang YL. The imprinted H19 gene regulates human placental trophoblast cell proliferation via encoding 
miR-675 that targets Nodal Modulator 1 (NOMO1).RNA Biol. 2012;9:1002-10.

9. Schmitz KJ, Helwig J, Bertram S, Sheu SY, Suttorp AC, Seggewiss J, Willscher E, Walz MK, Worm K, Schmid KW. Differential expression of microRNA-675, microRNA139-3p and microRNA-335 in benign and malignant adrenocortical tumours.J Clin Pathol. 2011;64:529-35.

10. Aygün $\mathrm{O}$, Mehta S, Grewal SI. HDAC-mediated suppression of histone turnover promotes epigenetic stability of heterochromatin.Nat Struct Mol Biol. 2013;20:547-54.

11. Figueiredo ML, Philip P, Stenberg P, Larsson J. HP1a recruitment to promoters is independent of $\mathrm{H} 3 \mathrm{~K} 9$ methylation in Drosophila melanogaster.PLoS Genet. 2012;8:e1003061.

12. Schuldt A. Chromatin: RNA eviction by HP1. Nat Rev Mol Cell Biol. 2012;13:478-9.

13. Lee YH, Kuo CY, Stark JM, Shih HM, Ann DK. HP1 promotes tumor suppressor BRCA1 functions during the DNA damage response.Nucleic Acids Res. 2013;41:578498.

14. Ayrapetov MK, Gursoy-Yuzugullu O, Xu C, Xu Y, Price BD. DNA double-strand breaks promote methylation of histone $\mathrm{H} 3$ on lysine 9 and transient formation of repressive chromatin.Proc Natl Acad Sci U S A. 2014;111:9169-74.

15. Zhang BL, Ni HB, Liu J, Lei Y, Li H, Xiong Y, Yao R, Yu ZQ, Gao DS. EGR1 participates in abnormally high gdnf gene transcription mediated by histone hyperacetylation in glioma cells.Biochim Biophys Acta. 2014;1839:1161-9.

16. Salotti J, Sakchaisri K, Tourtellotte WG, Johnson PF. An Arf-Egr-C/EBP $\beta$ Pathway Linked to Ras-Induced Senescence and Cancer.Mol Cell Biol. 2015;35:866-83.

17. Stoddart A, Fernald AA, Wang J, Davis EM, Karrison T, Anastasi J, Le Beau MM. Haploinsufficiency of del (5q) genes, Egr1 and Apc, cooperate with Tp53 loss to induce acute myeloid leukemia in mice.Blood. 2014;123:1069-78.

18. Kang HS, Ock J, Lee HJ, Lee YJ, Kwon BM, Hong SH. Early growth response protein 1 upregulation and nuclear translocation by 2'-benzoyloxycinnamaldehyde induces prostate cancer cell death.Cancer Lett. 2013;329:217-27.

19. Saayman S, Ackley A, Turner AM, Famiglietti M, Bosque A, Clemson M, Planelles V, Morris KV. An HIV-encoded antisense long noncoding RNA epigenetically regulates viral transcription. Mol Ther. 2014;22:1164-75.

20. Leighton PA, Saam JR, Ingram RS, Stewart CL, Tilghman SM.An enhancer deletion affects both H19 and Igf2 expression. Genes Dev. 1995, 9: 2079-89.

21. Bergström R, Whitehead J, Kurukuti S, Ohlsson R.CTCF regulates asynchronous replication of the imprinted $\mathrm{H} 19$ / Igf2 domain. Cell Cycle 2007, 6: 450-4.

22. Shoshani O, Massalha H, Shani N, Kagan S, Ravid O, Madar S, Trakhtenbrot L, Leshkowitz D, Rechavi G, Zipori D . Polyploidization of murine mesenchymal cells is associated with suppression of the long noncoding RNA
H19 and reduced tumorigenicity. Cancer Research 2012, 72: 6403-13.

23. Takeuchi S, Hofmann WK, Tsukasaki K.Loss of H19 imprinting in adult T-cell leukaemia/lymphoma. Br. J. Haematol. 2007, 137: 380-1.

24. Arima T, Matsuda T, Takagi N, Wake N .Association of IGF2 and H19 imprinting with choriocarcinoma development. Cancer Genet. Cytogenet. 1997, 93: 39-47

25. Banet G, Bibi O, Matouk I .Characterization of human and mouse H19 regulatory sequences. Mol. Biol. Rep. 2000, 27 : $157-65$.

26. Tanos V, Ariel I, Prus D, De-Groot N, Hochberg A .H19 and IGF2 gene expression in human normal, hyperplastic, and malignant endometrium. Int. J. Gynecol. Cancer 2004, 14:521-5.

27. Gao ZH, Suppola S, Liu J, Heikkilä P, Jänne J, Voutilainen $\mathrm{R}$.Association of $\mathrm{H} 19$ promoter methylation with the expression of H19 and IGF-II genes in adrenocortical tumors. J. Clin. Endocrinol. Metab. 2002, 87: 1170-6.

28. Lottin S, Adriaenssens E, Dupressoir T, Berteaux N, Montpellier C, Coll J, Dugimont T, Curgy JJ .Overexpression of an ectopic H19 gene enhances the tumorigenic properties of breast cancer cells. Carcinogenesis 2002, 23: 1885-95.

29. Barsyte-Lovejoy D, Lau SK, Boutros PC, Khosravi F, Jurisica I, Andrulis IL, Tsao MS, Penn LZ.The c-Myc oncogene directly induces the H19 noncoding RNA by allele-specific binding to potentiate tumorigenesis. Cancer Res. 2006, 66: 5330-7.

30. Li H, Yu B, Li J, Su L, Yan M, Zhu Z, Liu B.Overexpression of lncRNA H19 enhances carcinogenesis and metastasis of gastric cancer. Oncotarget. 2014;5:2318-29.

31. Matouk IJ, DeGroot N, Mezan S, Ayesh S, Abu-lail R, Hochberg A, Galun E.The H19 non-coding RNA is essential for human tumor growth. PLoS ONE 2007, 2: e845.

32. Ayesh S, Matouk I, Schneider T, Ohana P, Laster M, AlSharef W, De-Groot N, Hochberg A.Possible physiological role of H19 RNA. Mol. Carcinog. 2002, 35: 63-74.

33. Venkatraman A, He XC, Thorvaldsen JL, Sugimura R, Perry JM, Tao F, Zhao M, Christenson MK, Sanchez R, Yu JY, Peng L, Haug JS, Paulson A, et al. Maternal imprinting at the H19-Igf2 locus maintains adult haematopoietic stem cell quiescence.Nature. 2013;500:345-9.

34. Fujimori H, Mukai H, Murakami Y, Hemberger M, Hippo Y, Masutani M. The H19 induction triggers trophoblast lineage commitment in mouse ES cells.Biochem Biophys Res Commun. 2013;436:313-8.

35. Kallen AN, Zhou XB, Xu J, Qiao C, Ma J, Yan L, Lu L, Liu C, Yi JS, Zhang H, Min W, Bennett AM, Gregory RI, et al. The imprinted H19 lncRNA antagonizes let-7 microRNAs. Mol Cell. 2013;52:101-12.

36. Gao Y, Wu F, Zhou J, Yan L, Jurczak MJ, Lee HY, Yang L, Mueller M, Zhou XB, Dandolo L, Szendroedi J, Roden M, 
Flannery C, et al.The H19/let-7 double-negative feedback loop contributes to glucose metabolism in muscle cells. Nucleic Acids Res. 2014;42:13799-811.

37. Chen B, Yu M, Chang Q, Lu Y, Thakur C, Ma D, Yi Z, Chen F.Mdig de-represses H19 large intergenic noncoding RNA (lincRNA) by down-regulating H3K9me3 and heterochromatin. Oncotarget. 2013;4:1427-37.

38. Medrzycki M, Zhang Y, Zhang W, Cao K, Pan C, Lailler N, McDonald JF, Bouhassira EE, Fan Y. Histone h1.3 suppresses h19 noncoding RNA expression and cell growth of ovarian cancer cells.Cancer Res. 2014;74:6463-73).

39. Wong N, De Melo J, Tang D. PKM2, a Central Point of Regulation in Cancer Metabolism.Int J Cell Biol. 2013;2013:242513.

40. Luan W, Wang Y, Chen X, Shi Y, Wang J, Zhang J, Qian J, Li R, Tao T, Wei W, Hu Q, Liu N, You Y. PKM2 promotes glucose metabolism and cell growth in gliomas through a mechanism involving a let-7a/c-Myc/hnRNPA1 feedback loop. Oncotarget. 2015;6:13006-18.

41. Wang HJ, Hsieh YJ, Cheng WC, Lin CP, Lin YS, Yang SF, Chen CC, Izumiya Y, Yu JS, Kung HJ, Wang WC. JMJD5 regulates PKM2 nuclear translocation and reprograms HIF$1 \alpha$-mediated glucose metabolism.Proc Natl Acad Sci U S A. 2014;111:279-84.

42. Palsson-McDermott EM, Curtis AM, Goel G, Lauterbach MA, Sheedy FJ, Gleeson LE, van den Bosch MW, Quinn SR, Domingo-Fernandez R, Johnson DG, Jiang JK, Israelsen WJ, Keane J, et al. Pyruvate Kinase M2 Regulates Hif- $1 \alpha$ Activity and IL- $1 \beta$ Induction and Is a Critical Determinant of the Warburg Effect in LPS-Activated Macrophages.Cell Metab. 2015;21:65-80.

43. Hamabe A, Konno M, Tanuma N, Shima H, Tsunekuni K, Kawamoto K, Nishida N, Koseki J, Mimori K, Gotoh N, Yamamoto H, Doki Y, Mori M, et al. Role of pyruvate kinase M2 in transcriptional regulation leading to epithelialmesenchymal transition.Proc Natl Acad Sci U S A. 2014;111:15526-31.

44. Li L, Zhang Y, Qiao J, Yang JJ, Liu ZR. Pyruvate kinase M2 in blood circulation facilitates tumor growth by promoting angiogenesis.J Biol Chem. 2014;289:25812-21

45. Jung Y, Jang YJ, Kang MH, Park YS, Oh SJ, Lee DC, Xie Z, Yoo HS, Park KC, Yeom YI. Metabolic signature genes associated with susceptibility to pyruvate kinase, muscle type 2 gene ablation in cancer cells.Mol Cells. 2013;35:33541.

46. Keller KE, Doctor ZM, Dwyer ZW, Lee YS. SAICAR induces protein kinase activity of PKM2 that is necessary for sustained proliferative signaling of cancer cells.Mol Cell. 2014;53:700-9.

47. Israelsen WJ, Dayton TL, Davidson SM, Fiske BP, Hosios AM, Bellinger G, Li J, Yu Y, Sasaki M, Horner JW, Burga LN, Xie J, Jurczak MJ, et al. PKM2 isoform-specific deletion reveals a differential requirement for pyruvate kinase in tumor cells.Cell. 2013;155:397-409.
48. Desai S, Ding M, Wang B, Lu Z, Zhao Q, Shaw K, Yung WK, Weinstein JN, Tan M, Yao J. Tissue-specific isoform switch and DNA hypomethylation of the pyruvate kinase PKM gene in human cancers. Oncotarget. 2014;5:8202-10.

49. Jiang Y, Li X, Yang W, Hawke DH, Zheng Y, Xia Y, Aldape K, Wei C, Guo F, Chen Y, Lu Z.PKM2 regulates chromosome segregation and mitosis progression of tumor cells. Mol Cell. 2014;53:75-87.

50. Zhuang M, Gao W, Xu J, Wang P, Shu Y. The long noncoding RNA H19-derived miR-675 modulates human gastric cancer cell proliferation by targeting tumor suppressor RUNX1.Biochem Biophys Res Commun. 2014;448:315-22.

51. Tsang WP, Ng EK, Ng SS, Jin H, Yu J, Sung JJ, Kwok TT. Oncofetal H19-derived miR-675 regulates tumor suppressor RB in human colorectal cancer.Carcinogenesis. 2010 31:350-8.

52. Hernandez JM, Elahi A, Clark CW, Wang J, Humphries LA, Centeno B, Bloom G, Fuchs BC, Yeatman T, Shibata D. miR-675 mediates downregulation of Twist 1 and $\mathrm{Rb}$ in AFP-secreting hepatocellular carcinoma.Ann Surg Oncol. 2013;20 Suppl 3:S625-35.

53. Eissenberg JC, Elgin SC. HP1a: a structural chromosomal protein regulating transcription. Trends Genet. 2014;30:10310.

54. Azzaz AM, Vitalini MW, Thomas AS, Price JP, Blacketer MJ, Cryderman DE, Zirbel LN, Woodcock CL, Elcock AH, Wallrath LL, Shogren-Knaak MA. Human heterochromatin protein $1 \alpha$ promotes nucleosome associations that drive chromatin condensation.J Biol Chem. 2014;289:6850-61.

55. Bracken AP, Pasini D, Capra M, Prosperini E, Colli E, Helin K. EZH2 is downstream of the pRB-E2F pathway, essential for proliferation and amplified in cancer.EMBO J. 2003;22:5323-35.

56. Canzio D, Liao M, Naber N, Pate E, Larson A, Wu S, Marina DB, Garcia JF, Madhani HD, Cooke R, Schuck P, Cheng Y, Narlikar GJ. A conformational switch in HP1 releases auto-inhibition to drive heterochromatin assembly. Nature. 2013;496:377-81.

57. Ma C, Nong K, Zhu H, Wang W, Huang X, Yuan Z, Ai K. H19 promotes pancreatic cancer metastasis by derepressing let-7's suppression on its target HMGA2-mediated EMT. Tumour Biol. 2014;35:9163-9.

58. Guo G, Kang Q, Chen Q, Chen Z, Wang J, Tan L, Chen JL. High expression of long non-coding RNA H19 is required for efficient tumorigenesis induced by Bcr-Abl oncogene. FEBS Lett. 2014;588:1780-6.

59. Luo M, Li Z, Wang W, Zeng Y, Liu Z, Qiu J. Long noncoding RNA H19 increases bladder cancer metastasis by associating with EZH2 and inhibiting E-cadherin expression.Cancer Lett. 2013;333:213-21.

60. Zhang L, Yang F, Yuan JH, Yuan SX, Zhou WP, Huo XS, $\mathrm{Xu}$ D, Bi HS, Wang F, Sun SH. Epigenetic activation of the MiR-200 family contributes to H19-mediated metastasis 
suppression in hepatocellular carcinoma.Carcinogenesis. 2013;34:577-86.

61. Shoshani O, Massalha H, Shani N, Kagan S, Ravid O, Madar S, Trakhtenbrot L, Leshkowitz D, Rechavi G, Zipori D. Polyploidization of murine mesenchymal cells is associated with suppression of the long noncoding RNA H19 and reduced tumorigenicity.Cancer Res. 2012;72:640313.

62. Yang F, Bi J, Xue X, Zheng L, Zhi K, Hua J, Fang G. Up-regulated long non-coding RNA H19 contributes to proliferation of gastric cancer cells.FEBS J. 2012 279:315965.

63. Cortés-Cros M, Hemmerlin C, Ferretti S, Zhang J, Gounarides JS, Yin H, Muller A, Haberkorn A, Chene P, Sellers WR, Hofmann F. M2 isoform of pyruvate kinase is dispensable for tumor maintenance and growth.Proc Natl Acad Sci U S A. 2013;110:489-94.

64. Wong CC, Au SL, Tse AP, Xu IM, Lai RK, Chiu DK, Wei LL, Fan DN, Tsang FH, Lo RC, Wong CM, Ng IO.Switching of pyruvate kinase isoform $\mathrm{L}$ to $\mathrm{m} 2$ promotes metabolic reprogramming in hepatocarcinogenesis. PLoS One. 2014;9:e115036.

65. Yang W, Xia Y, Hawke D, Li X, Liang J, Xing D, Aldape K, Hunter T, Alfred Yung WK, Lu Z. PKM2 phosphorylates histone $\mathrm{H} 3$ and promotes gene transcription and tumorigenesis. Cell. 2012 17;150:685-96.

66. Yang W, Zheng Y, Xia Y, Ji H, Chen X, Guo F, Lyssiotis CA, Aldape K, Cantley LC, Lu Z. ERK1/2-dependent phosphorylation and nuclear translocation of PKM2 promotes the Warburg effect.Nat Cell Biol. 2012;14:1295304.

67. Chen $\mathrm{CL}, \mathrm{Wu} \mathrm{JC}$, Chen GY, Yuan PH, Tseng YW, Li KC, Hwang SM, Hu YC. Baculovirus-mediated miRNA regulation to suppress hepatocellular carcinoma tumorigenicity and metastasis. Mol Ther 2015;23:79-88. 